\title{
Mechanisms of Action of Autophagy Modulators Dissected by Quantitative Systems Pharmacology Analysis
}

\author{
Qingya Shi 1,2, Fen Pei ${ }^{1}$, David H. Perlmutter ${ }^{3}$, Bing Liu 1,* , and Ivet Bahar 1,* \\ 1 Department of Computational and Systems Biology, School of Medicine, University of Pittsburgh, PA, \\ 15213, USA; qingya@pitt.edu (Q.S.); fep7@pitt.edu (F.P.) \\ 2 School of Medicine, Tsinghua University, Beijing, 100084, China \\ 3 Department of Pediatrics, School of Medicine, Washington University in St. Louis, MO, 63130, USA; \\ perlmutterd@wustl.edu (D.P.) \\ * Correspondence: liubing@pitt.edu (B.L.); bahar@pitt.edu (I.B.)
}

Received: date; Accepted: date; Published: date

\begin{abstract}
Autophagy plays an essential role in cell survival/death and functioning. Modulation of autophagy has been recognized as a promising therapeutic strategy against diseases/disorders associated with uncontrolled growth or accumulation of biomolecular aggregates, organelles or cells including those caused by cancer, aging, neurodegeneration, and liver diseases such as $\alpha 1$ antitrypsin deficiency. Numerous pharmacological agents that enhance or suppress autophagy have been discovered. However, their molecular mechanisms of action are far from clear. Here we collected a set of 225 autophagy modulators and carried out a comprehensive quantitative systems pharmacology (QSP) analysis of their targets using both existing databases and predictions made by our machine learning algorithm. Autophagy modulators include several highly promiscuous drugs (e.g. artenimol and olanzapine acting as activator, fostamatinib as inhibitor, or melatonin as dual-modulator), as well as selected drugs uniquely targeting specific proteins $(\sim 30 \%$ of modulators). They are mediated by three layers of regulation: (i) pathways involving core autophagy-related (ATG) proteins such as mTOR, AKT, and AMPK; (ii) upstream signaling events that regulates the activity of ATG pathways such as calcium-, cAMP-, and MAPK-signaling pathways; and (iii) transcription factors regulating the expression ATG proteins such as TFEB, TFE3, HIF-1, FoxO, and NF- $\kappa B$. Our results suggest that PKA serves as a linker bridging between various signal transduction events and autophagy. These new insights contribute to a better assessment of the mechanism of action of autophagy modulators as well as their side effects, development of novel polypharmacological strategies, and identification of drug repurposing opportunities.
\end{abstract}

Keywords: autophagy; quantitative systems pharmacology; signal transduction; machine learning; drug-target interactions; mechanism of action; mTOR; PKA; PI3K

\section{Introduction}

Autophagy is the process that enables self-clearance of potentially damaging aggregates, dysfunctional organelles (e.g. mitochondria by mitophagy) or entire cells, and as such it is one of the most significant regulatory processes that ensure healthy functioning of cells [1,2]. During starvation, autophagy can serve as an essential source of energy for survival, and in general it plays a housekeeping role to maintain the homeostasis and viability of the cells [3]. In addition, autophagy has been shown to be instrumental in erasing pathological responses or maintaining physiological functions of the cells such as anti-aging, tumor suppression and antigen presentation [4].

Once autophagy is activated, e.g. by stress stimuli such as starvation, a cascade of reactions are triggered that lead to the formation a double-membrane-bound vesicle called autophagosome [5], which encapsulates the cytoplasmic aggregates or dysfunctional organelles. The subsequent fusion of the autophagosome with the lysosome to form the autolysosome permits the lysosomal enzymes to digest and degrade the contents of the autophagosome $[5,6]$. 
According to our current understanding, autophagic responses are modulated by many events, in which the kinase mammalian target of rapamycin (mTOR) plays a major role as a master regulator of autophagy [7]. mTOR, a serine/threonine kinase, is a downstream regulator of the phosphoinositide 3-kinase (PI3K) pathway. It suppresses autophagy induction by inhibiting the activation of the UNC-5 like autophagy activating kinase (ULK) complex [8]. The Atg1/ULK complex otherwise receives signals on cellular nutrient status and recruits downstream autophagy-related (ATG) proteins to the autophagosome formation site to initiate the autophagic response [9]. mTOR further acts as a negative regulator of autophagy by preventing the general expression of lysosomal and autophagy genes through phosphorylating transcription factor EB (TFEB) $[7,8]$.

Other important regulators of autophagy include cAMP-dependent protein kinase A (PKA) [10] and other proteins involved in $\mathrm{Ca}^{2+}$ signaling pathways. $\mathrm{Ca}^{2+}$ ions have complex effects $[11,12]$ : cytoplasmic $\mathrm{Ca}^{2+}$ activates $\mathrm{Ca}^{2+} /$ calmodulin-dependent kinase kinase $\beta(\mathrm{CaMKK} \beta)$, which activates AMP-activated protein kinase (AMPK) to promote autophagy [13]. But CaMKK $\beta$ also activates calpain, which inhibits autophagy by cleaving autophagy related 5 (Atg5) protein [14].

In the last few decades, there has been a surge in the number of autophagy-modulating drugs targeting these well-known autophagy regulators. For example, rapamycin which inhibits mTOR is known as one of the most important autophagy activators [15], and wortmannin which inhibits PI3K is known as a significant autophagy inhibitors $[16,17]$. In the meantime, autophagy modulation has been receiving increased attention as a strategy for developing therapeutics [18]. Autophagy has been widely accepted as cytoprotective against neurodegenerative diseases and many clinical interventions are moving forward to increase autophagy as a therapeutic intervention [19]. For example, rapamycin is regarded as an important pharmacological agent against Alzheimer's disease (AD) [20]. Various autophagy-enhancing drugs (such as carbamazepine [21], fluphenazine [22], and glibenclamide [23]) have been identified to have therapeutic effects against $\alpha 1$ antitrypsin deficiency (ATD) caused by the aggregation of the misfolded $\alpha 1$-antitrypsin Z (ATZ) proteins in the liver. Besides, autophagy dysfunction is also highly associated with cancer, and many autophagy-targeting drugs also serve as anticancer drugs [24]. For instance, metformin, an autophagy enhancer, is a drug against myeloma [1].

In this study, we carried out a comprehensive analysis of a set of 225 known autophagy modulators selected from DrugBank v5.1.1 [25] (Supplementary Table S1), their 993 target proteins, and 294 associated pathways in order to obtain a systems-level understanding of the common mechanisms of autophagy modulation, as well as specific mechanisms of selected modulators. The set of modulators consists of 174 activators, 31 inhibitors, and 20 compounds acting as dualmodulators. We identified 1,831 known interactions between these drugs and their targets, and predicted 368 novel interactions using our probabilistic matrix factorization (PMF)-based application programming interface [26-28]. Of these predictions, 75 were consistent with recently published experimental data (not yet deposited in DrugBank [25]).

Our results show that autophagy modulators are structurally diverse and functionally promiscuous. Their target proteins are commonly expressed in the brain and liver tissues and mainly function as tyrosine kinases, calcium channels, aminergic GPCRs, or nuclear hormone receptors. The most frequently targeted proteins do not directly take part in autophagy pathway, but indirectly regulate the activity/expression level of core ATG proteins through differential signaling pathways. Through GO annotation and pathway enrichment analysis, we distinguished the most important signaling pathways that regulate autophagy. Our results strongly point to the crucial role of PKA as a hub conveying autophagy signals, and directly regulating ATG proteins and their gene expression.

\section{Results}

\subsection{Autophagy Modulators Are Structurally Diverse and Have Promiscuous Functions}

We manually curated the role of the selected 225 autophagy modulators based on the literature, and classified them into three functional categories: activators $(n=174)$, inhibitors $(n=31)$, and dualmodulators $(n=20)$. Here 'dual-modulators' refer to drugs that can both positively and negatively regulate autophagy depending on the biological context. For instance, melatonin is a dual-modulator 
because it reduces autophagic activity in tumor trophoblast cells while it enhances it in normal cells [29].

As a first step we examined to what extent the autophagy modulators within each group share structural and functional similarities. Supplementary Figure S1 shows that the selected autophagy modulators are highly diverse. Within all three categories, most of the drug pairs have low structural and functional similarities $(<0.5)$ evaluated based on their 2D fingerprints and drug-target interaction patterns extracted from DrugBank, respectively. Yet, we can distinguish clusters of drugs that have similar structures, which also share similar interaction patterns with targets. For example, rapamycin and its derivatives everolimus, temsirolimus, and ridaforolimus (Supplementary Figure S1a, activators \#28-30, enclosed in yellow ellipse) activate autophagy by inhibiting mTOR [30], and retain the same structure, presumably targeting the same site on mTOR. In contrast, the upper right white region on the right map (drugs \#141-166) indicates several autophagy activators (e.g. fluphenazine, pimozide, clonidine, paroxetine, triflupromazine, chlorpromazine, citalopram, nortriptyline, fluspirilene, doxazosin, amiodarone, flunarizine, verapamil, and dronedarone) that share similar interaction patterns. Most of them are therapeutic agents for mental disorders such as schizophrenia, depression, and anxiety disorders, or for cardiovascular diseases such as high blood pressure, angina and arrhythmia, regulating autophagy through cAMP- and $\mathrm{Ca}^{2+}$-signaling pathways. Yet, the left map indicates that they are structurally heterogeneous, suggesting that they have different targets on their shared pathways.

Further examination of Supplementary Figure S1 shows that macrolide antibiotics erythromycin, clarithromycin, azithromycin (inhibitors \#5-7 in panel b) inhibit autophagy via ER stress-mediated C/EBP homologous protein (CHOP) induction [31], but despite their close structural similarity (see enclosed region in panel $\mathbf{b}$, left), they exhibit distinctive interaction patterns with their targets (panel $\mathbf{b}$, right), suggesting different mechanisms of action. Phenothiazine antipsychotics thioridazine and trifluoperazine (Supplementary Figure S1c, dual-modulators \#2-3) regulate autophagy through dopamine receptors [32] and share close structural similarities.

\subsection{Selected Autophagy Modulators Are Distinguished by Their High Promiscuity}

The space of proteins targeted by autophagy modulators is quite broad. We identified 993 such proteins (Supplementary Table S2) composed of 706 targets associated with the 174 activators, 374 associated with the 31 inhibitors, and 94 targets associated with the 20 dual-modulators, which show partial overlaps, as presented by the Venn diagram in Figure 1a (right). The space of drugs and targets may be viewed as a bipartite network, with multiple connections (drug-target interactions). The number of targets connected to a given modulator will be referred to as the degree of the modulator. The higher the degree, the more promiscuous the modulator.

Based on DrugBank v5.1.1, we identified a total of 1,831 types of known drug-target interactions between these autophagy modulators and their target proteins (Supplementary Table S3). Specifically, the activators, inhibitors and dual-modulators were involved in 1339, 389 and 103 known interactions with their targets (Figure 1a). 
a

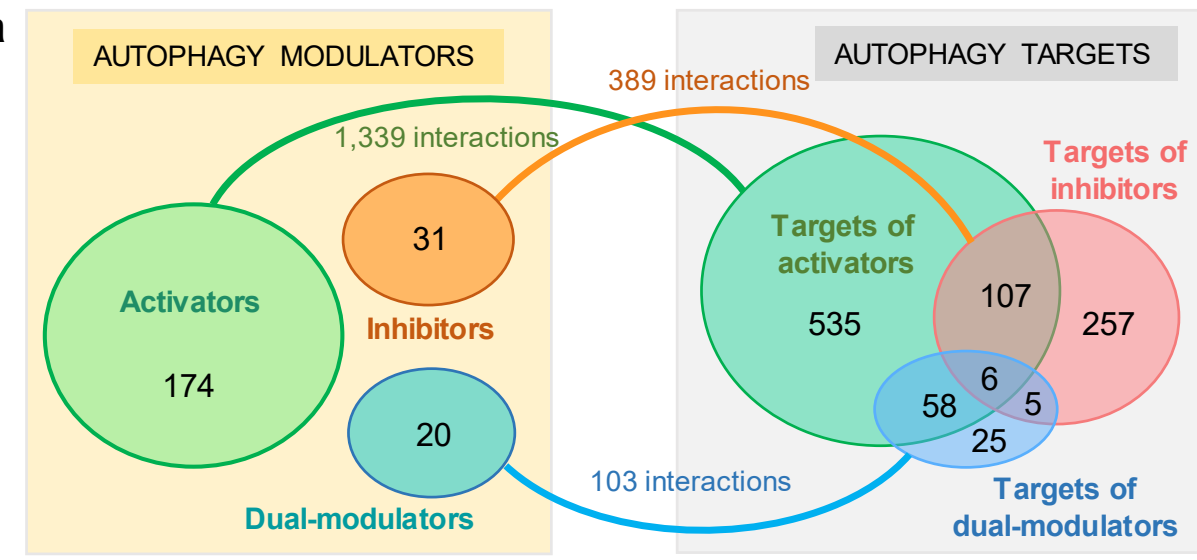

b

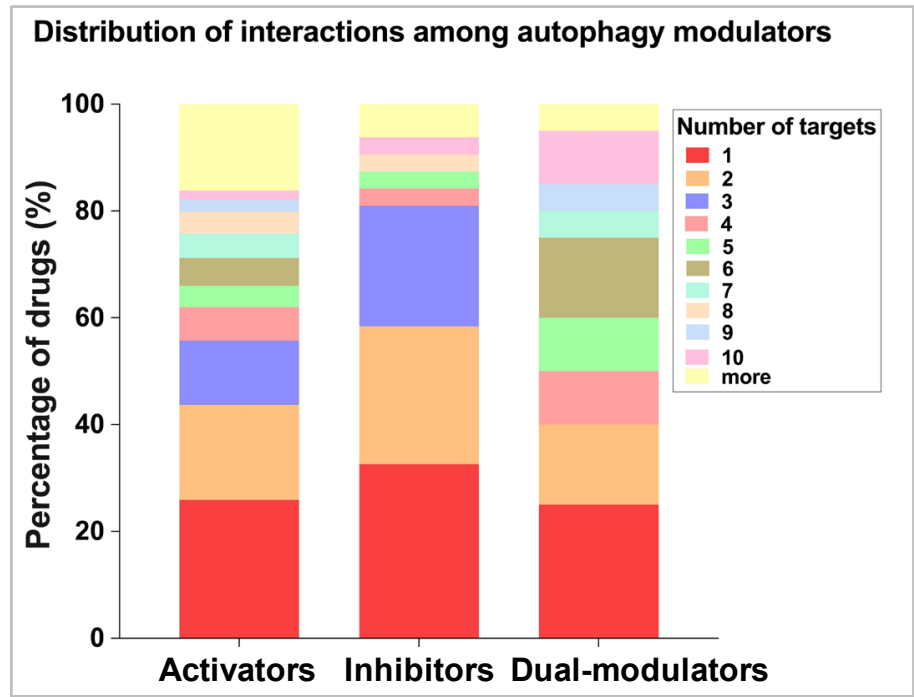

Figure 1. Space of autophagy modulators and their targets. (a) On the left we display three groups of autophagy modulators, and on the right the corresponding targets, which show considerable overlaps. The number of targets in each subset are shown by the labels in the Venn diagrams on the right. The numbers on the linkers connecting the drugs to the targets indicate the number of targets associated with each category of autophagy modulators; (b) Distribution of the degrees (number of associated targets) for each category of autophagy modulators.

In principle, if each autophagy modulator had one distinctive target, we would have 225 targets (or even less as some targets could overlap), but this is not the case: several modulators are highly promiscuous (Supplementary Figure S3), in the sense that they interact with multiple targets. The detailed distribution of the multiplicity of targets among modulators is summarized in Figure $\mathbf{1 b}$. Only $25-32 \%$ of the modulators interact with a single drug (red portions of the bars), depending on the type of modulator. It is interesting to note that almost $20 \%$ of activators have more than 10 targets (yellow portion at the top of the leftmost bar), pointing to the high promiscuity of $\sim 30$ activators. Precisely, 31 out of 174 (17.8\%) activators, 3 out of 31 (9.7\%) inhibitors, and 3 out of 20 (15.0\%) dualmodulators have at least 10 known targets.

Figure 2 provides more details on the identity of specific modulators with high promiscuity. The ordinates show the number of targets for autophagy activators (panel a), and all autophagy inhibitors (panel b) and dual-modulators (panel c), and the abscissa display the modulators reordered in decreasing promiscuity. See also Supplementary Figure S2 for the target number distribution of additional (less promiscuous) activators. We observe an average of 7.7 interactions per activator, 2.9 per inhibitor (if we exclude one outlier, fostamatinib; see below), and 5.3 per dual-modulator. The insets in the three panels of Figure 2 shows the histograms of modulators with different promiscuity. 
bioRxiv preprint doi: https://doi.org/10.1101/2020.03.25.007344; this version posted March 25, 2020. The copyright holder for this preprint (which was not certified by peer review) is the author/funder, who has granted bioRxiv a license to display the preprint in perpetuity. It is made available under aCC-BY 4.0 International license.

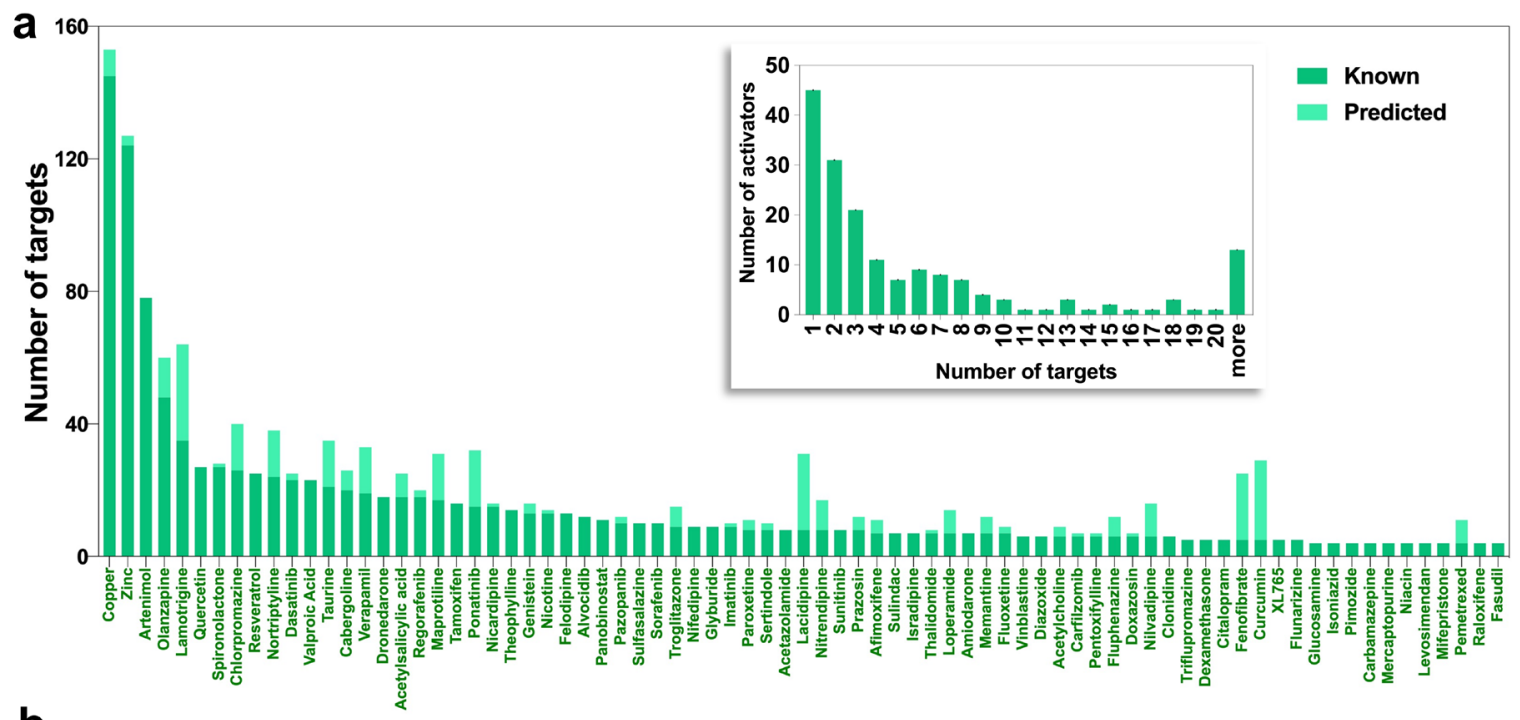

b
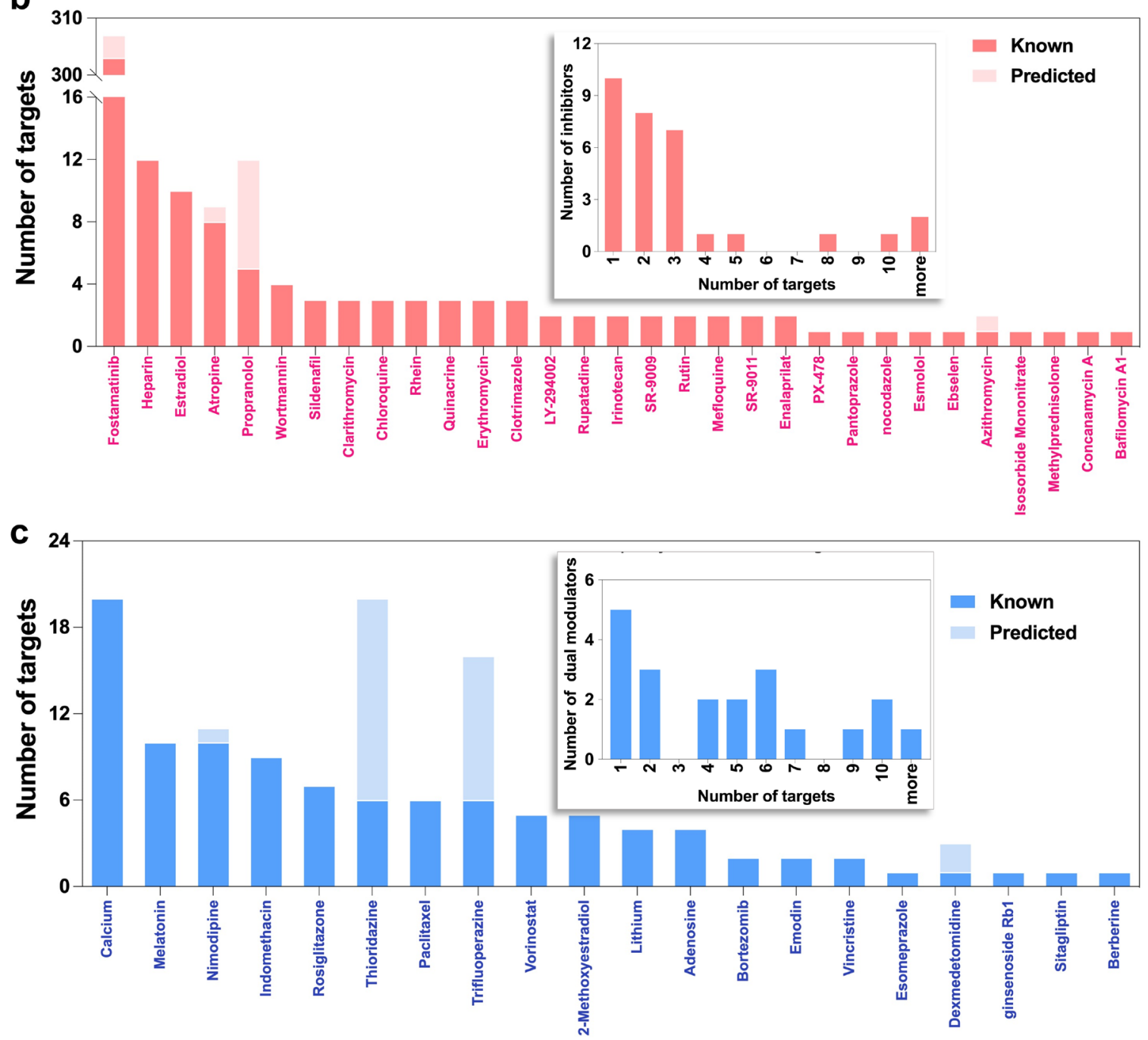

Figure 2. Promiscuity of autophagy modulators. (a) The number of known and predicted targets of activators; (b) The number of known and predicted targets of inhibitors; (c) The number of known and predicted targets of dual-modulators. The drugs are ranked in a descending order of the number of known targets. The contributions of the known and predicted drugs to the total number of targets is shown by the dark and light colors as indicated by the labels. See also Supplementary Figure S2 for additional data on activators. Insets: Number distribution of modulators vis-à-vis their promiscuity. The promiscuity is quantified by the degree of each modulator, i.e. the number of targets (abscissa) associated with the modulator. See also percent distributions in Supplementary Figure S4. 
Notably, of the 1,339 interactions in which the activators are involved, 145 are made by the most promiscuous activator, copper (Figure 2a, leftmost bar), followed by zinc (124 interactions/targets), artenimol (78 targets), and olanzapine (48 targets). Transition metal copper and zinc are nonstructural intracellular signaling mediators and essential elements of many enzymes. They both can induce autophagy by activating kinases such as mitogen-activated protein kinase (MAPK) [33,34]. A recent study shows that copper can even directly bind to ULK1/2 to enhance its pro-autophagic activity [35]. Antimalarial drug artenimol is a derivative of artemisinin that effectively kills $P$. falciparum by producing reactive oxygen [36] and targeting multiple biological processes otherwise essential for parasite survival [37]. The oxidative stresses induced by artemisinin and its derivatives also stimulate autophagy in the host cells $[38,39]$. As an antipsychotic drug, olanzapine mainly targets receptors of neurotransmitters such as dopamine, serotonin (5-HT), and $\gamma$-aminobutyric acid (GABA), which have been reported to regulate autophagy [32,40,41]. Further, olanzapine also induces autophagy by activating AMP-activated protein kinase (AMPK) signaling [42,43] and up-regulating the expression of the products of ATG genes such as ATG5 and Beclin-1 [44].

The most promiscuous autophagy inhibitor is fostamatinib (303 targets). Fostamatinib is an FDA-approved drug for the treatment of chronic immune thrombocytopenia. It has been reported that fostamatinib suppresses autophagy by inhibiting the spleen tyrosine kinase (STK) [45]. Fostamatinib also inhibits critical autophagy regulators including ULK1/2, serine/threonine-protein kinase (TBK1) [46], death-associated protein kinase 1 and 2 (DAPK1/2) [47,48], and leucine-rich repeat serine/threonine-protein kinase 2 (LRRK2) [49].

The most promiscuous dual-modulators of autophagy is calcium (20 targets), followed by melatonin (10 targets). $\mathrm{Ca}^{2+}$ is a cofactor of many enzymes and also acts as a second messenger mediating various signal transduction pathways. It targets calcium channels (e.g. $\mathrm{Ca}^{2+}$ voltagedependent channel subunit $\alpha 1 \mathrm{c}, \mathrm{CACNA1C}$ ), calcium-transporting ATPase (e.g. ATP2C1), and downstream calcium-binding proteins (e.g. calmodulin). Cytoplasmic $\mathrm{Ca}^{2+}$ inhibits autophagy via activation of calpain and inositol trisphosphate receptor $\left(\mathrm{IP}_{3} \mathrm{R}\right)$, while it also enhances autophagy via activation of AMPK [11]. Our previous study suggested that $\mathrm{Ca}^{2+} /$ calmodulin-dependent kinase kinase $\beta(\mathrm{CaMKK} \beta)$ plays a key role in regulating the balance between these opposing actions and acts as a determinant of the effect of competing roles of cytoplasmic $\mathrm{Ca}^{2+}$ in autophagy regulation [11].

To further identify potential drug-target interactions in DrugBank, we used our PMF model (see Materials and Methods) for predicting new targets for autophagy modulators. This analysis led to 368 novel interactions with high confidence scores $(>0.6)$, which included 12 additional targets. These pairs (not reported in the DrugBank) are listed in the Supplementary Table S4. The number of predicted targets for each drug are shown in Figure 2 by the light-shaded portions of the bars. Notably, 75 (out of 368) predicted pairs are consistent with already published experimental data [50-52]. Table 1 lists 20 of them, along with the corresponding experimentally measured binding affinities; and others can be seen from the entries in Supplementary Table S4 where experimental binding affinities are listed. For example, the promiscuous drugs olanzapine and fostamatinib mentioned earlier were found to bind to serotonin receptor 1F (HTR1F) and fibroblast growth factor receptor 4 (FGFR4), respectively. Fluphenazine, fluspirilene, thioridazine, sertindole, and trifluoperazine were identified to bind to dopamine receptor D3 (DRD3), and experiments indeed indicate their high affinities $\left(K_{i}<\right.$ $5 \mathrm{nM}$ ). We also detected various drug-target interactions with modest-to-weak affinities (e.g. the interaction between imatinib and Fms related receptor tyrosine kinase 3 (FLT3)).

Taken together, the 225 autophagy modulators we selected are diverse in terms of both structure and function. Several autophagy modulators, and in particular autophagy activators, are distinguished by their highly promiscuous effects. The additional drug-target interactions we identified using the PMF model are plausible, thus complementing the existing knowledge, and also helping generate new hypotheses that await further testing. To have a better understanding of the effects of autophagy modulators, we next analyze their target proteins. 
Table 1. Experimental validation of predicted drug-target interactions.

\begin{tabular}{llcc}
\hline Drug & Predicted Target ${ }^{1}$ & Binding affinity $\boldsymbol{K}_{\boldsymbol{i}}(\mathbf{n M})$ & Reference \\
\hline olanzapine & HTR1F & 310 & {$[52]$} \\
fostamatinib & FGFR4 & 350 & {$[50]$} \\
fluphenazine & DRD3 & 0.11 & {$[52]$} \\
fluspirilene & DRD3 & 0.40 & {$[52]$} \\
thioridazine & DRD3 & 1.5 & {$[52]$} \\
sertindole & DRD3 & 2.5 & {$[51]$} \\
trifluoperazine & DRD3 & 4.2 & {$[51]$} \\
prazosin & HDRA2C & 10.7 & {$[52]$} \\
thioridazine & HRH1 & 16 & {$[52]$} \\
chlorpromazine & CHRM5 & 42 & {$[52]$} \\
sorafenib & PDGFRA & 62 & {$[50]$} \\
pimozide & HTR1A & 88 & {$[52]$} \\
verapamil & HTR2A & 140 & {$[52]$} \\
fluoxetine & HTR2A & 148 & {$[52]$} \\
maprotiline & DRD1 & 402 & {$[52]$} \\
propranolol & HTR2C & 574 & {$[52]$} \\
nortriptyline & HRH2 & 645 & {$[52]$} \\
acetylcholine & CHRM5 & 800 & {$[52]$} \\
dasatinib & FGFR2 & 1400 & {$[50]$} \\
imatinib & FLT3 & 6300 & {$[50]$} \\
\hline${ }^{1}$ Full names of targets are given in Supplementary Table S4. See other experimentally validated predictions in \\
Supplementary Table S4 (rows with binding affinity information in the last column). &
\end{tabular}

\subsection{Frequent Targets of Autophagy Modulators Are Not ATG Proteins But Their Regulators}

As described above, the set of autophagy modulators have 1,005 targets (993 known/reported in DrugBank plus 12 identified here; see Supplementary Table S3). While drugs were highly promiscuous, the targets usually had smaller number of connections to drugs in the bipartite network representing drug-target pairs. We observed an average of 1.9 interactions per target; and $66.9 \%$ of the targets interacted with one single drug.

We show in Figure 3a the most frequently targeted proteins (51 of them), which interact with five or more known autophagy modulators. The bars display the corresponding numbers of modulators broken down by color-coded categories, with dark and light shaded portions referring to known and predicted interactions, respectively. We refer to this set of proteins as 'frequent targets', FTs, of autophagy modulators. The distribution of autophagy targets over protein families, extracted from family memberships given in Uniprot [53] is presented in Figure 3b. Kinases (32.17\%), ion channels (6.76\%, including ligand-gated ion channels, $\mathrm{Ca}^{2+}$ channels and $\mathrm{Na}^{+}$channels), and $\mathrm{G}$ protein coupled receptors (GPCRs) (5.85\%) are three major protein families targeted by our autophagy modulators. However, this presumably reflects the historical dominance of these families among drug target, noted in earlier studies [54]. More meaningful are the identities of the specific targets belonging to these families, which are analyzed next in relation to ATG proteins.

In order to see whether FTs overlap with the targets involved in autophagy pathways, we compared them to ATG proteins participating in the canonical autophagy pathway (KEGG id: hsa04140). Thirty-two out of 1,005 targets of autophagy modulators were found to be ATG proteins, including mTOR, ULK1/2, AMPK, CAMKK $\beta$, insulin (INS), AKT, PI3K, PKA, PKC, phosphoinositide-dependent kinase 1 (PDK1), Raf1, ERK1/2, JNK2/3, Beclin-1, BCL2, DAPK1/2/3, cathepsin L (CTSL), inositol-requiring enzyme 1 (IRE1), eukaryotic translation inhibition factor $2 \alpha$ kinase 4 (EIF2AK4), hypoxia inducible factor $1 \alpha$ (HIF-1 $\alpha$ ) (see the rows highlighted in green in Supplementary Table S2). Among them, 15 are targeted by autophagy activators, 26 by inhibitors, and two by dual-modulators. Among the ATG proteins, only mTOR is a FT. This analysis therefore showed that the majority of ATG proteins are not FTs of autophagy modulators. 
a

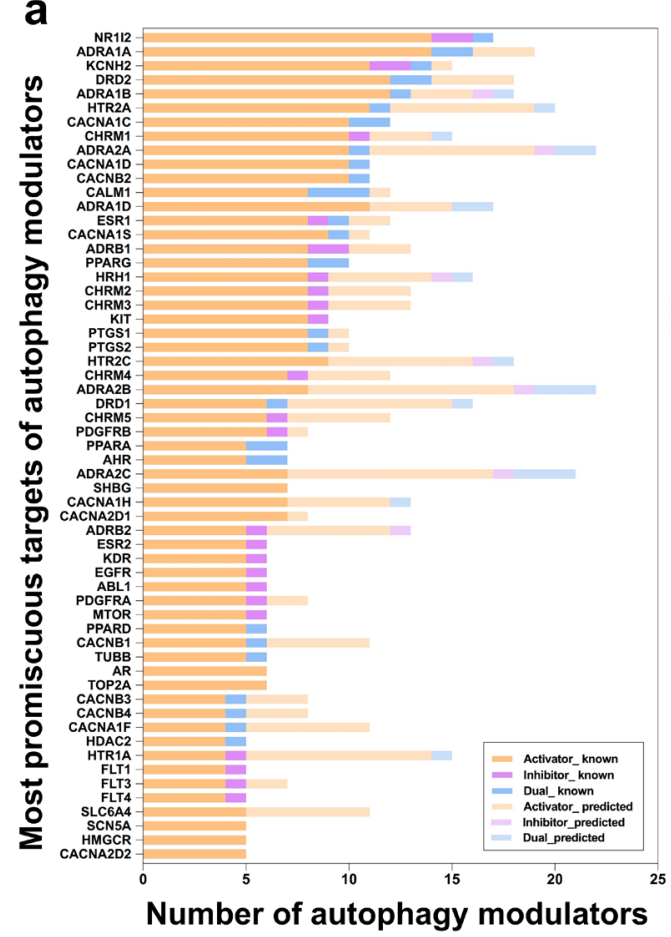

b cytochrome family (1.41\%)

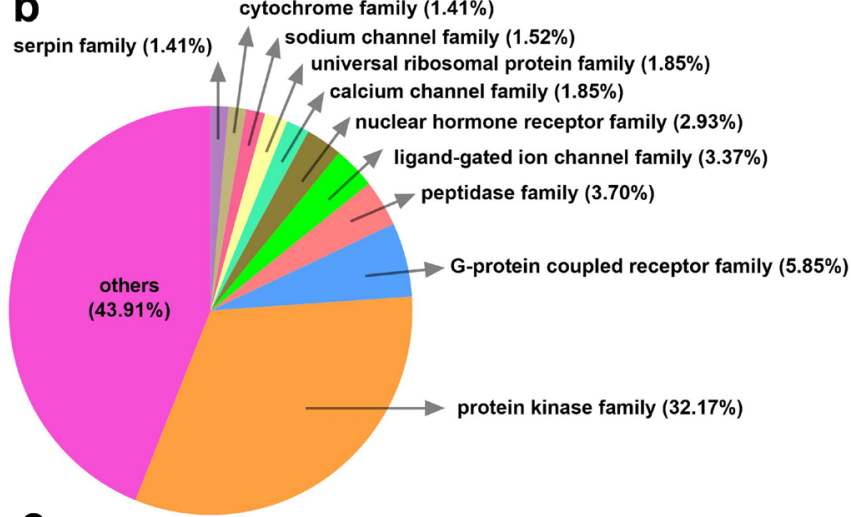

C

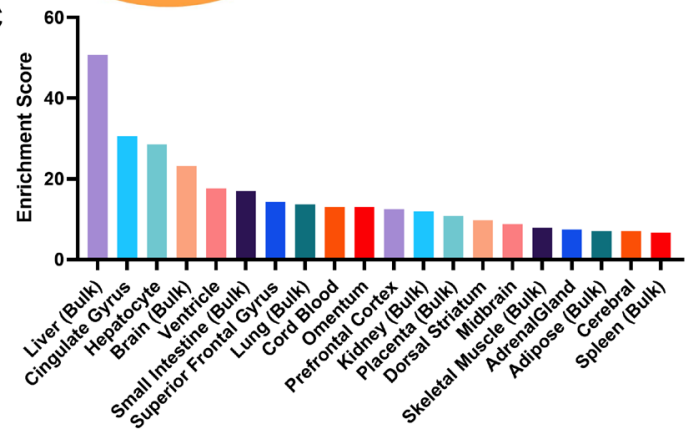

Figure 3. An overview of proteins targeted by autophagy modulators. (a) Frequent targets of autophagy activators (orange), inhibitors (red), and dual-modulators (blue), ranked by the number of the total known targets in descending order. In light shades are those predicted by our PMF method. (b) Distribution of targeted proteins by protein families; (c) Tissues enriched in targets of autophagy modulators

The question was then to assess what the FTs are, and why they are the targets of many autophagy modulators. We conduct next a more detailed analysis of the FTs.

Figure 3a shows that a nuclear hormone receptor (NHR) (subfamily 1 group I member 2 (NR1I2) is the most frequently targeted protein NR1I2 is found here to interact with 14 activators, one inhibitor, and one dual-modulator. NR1I2 gene encodes the pregnane $X$ receptor that regulates the transcription of many genes involved in the metabolism and secretion of potentially harmful endogenous and xenobiotic compounds, and in the homeostasis of glucose, lipid, cholesterol, bile acid, and bilirubin. The current analysis strongly suggests that it also activates the genes involved in autophagic elimination. There are six more NHRs that are FTs: estrogen receptor 1 and 2 (ESR1, ESR2), androgen receptor (ANDR), and peroxisome proliferator activated receptors $\gamma, \alpha$, and $\delta$ (PPARG, PPARA, PPARD). Numerous studies have reported that these transcription factors regulate the expression of core ATG proteins. Specifically, ESR1 and ESR2 regulate mTOR expression (see review [55]). ANDR regulates the transcription of ULK1/2, ATG4B/D, and transcription factor EB (TFEB), a master regulator of autophagy genes [56]. PPARs are known to regulate autophagy via AMPK and phosphatase and tensin homolog (PTEN) expression [57].

Eleven FTs directly participate in calcium signaling, including calmodulin and 10 ion channel family members: $\mathrm{Ca}^{2+}$ voltage-gated channel subunit $\alpha 1 \mathrm{c}$ (CACNA1C) and other members of the same family (CACNA1D, CACNB2, CACNA1S, CACNA1H, CACNA2D1, CACNB1, CACNA2D2, $\mathrm{K}^{+}$voltage-gated channel subfamily $\mathrm{H}$ member $2(\mathrm{KCNH} 2)$, and $\mathrm{Na}^{+}$voltage-gated channel $\alpha$ subunit 7 (SCN6A). We note that calmodulin interacts with 9 activators and three dual-modulators, consistent with the complex role of cytoplasmic $\mathrm{Ca}^{2+}$ in regulating autophagy.

Eighteen FTs are GPCR family members, consisting of $\alpha 1 \mathrm{~A}$ adrenergic receptor ADRA1A and its family members (ADRA1B, ADRA1D, ADRB1, ADRB2, ADRA2A, ADRA2B, ADRA2C), dopamine receptors (DRD1, DRD2), histamine receptor HRH1, serotonin receptors (HTR2A, HTR2C) and muscarinic receptors (CHRM1-5). GPCRs regulate autophagy through diverse downstream signaling pathways [58]. For example, $\alpha 1$-adrenergic receptor (ADRA1) activation induces protein kinase B (AKT)-mediated autophagy. $\beta$-adrenergic receptors (ADRB1 and ADRB2) promote 
autophagy via cAMP pathway [59]. CHRM3 regulates autophagy via CaMKK $\beta$-AMPK pathway [60]. DRD1 stimulation induces autophagy via a cAMP-dependent but EPAC-independent mechanism [61], while D2-like family of dopamine receptors (DRD2-4) positively regulate autophagy through AKT-mMOR and AMPK pathways [51,62]. Furthermore, activation of HTR2 inhibits autophagy via mTOR-independent p70S6K and 4E-EP1 phosphorylation [63].

As shown in Figure $3 b, 32.17 \%$ of the targets are protein kinases mediating intracellular signal transduction. Among them, seven are FTs, including mTOR and several receptor tyrosine kinases (RTKs): epidermal growth factor receptor (EGFR), vascular endothelial growth factor receptor 2 (KDR), Abelson murine leukemia viral oncogene homolog 1 (ABL1), proto-oncogene c-KIT (KIT), and platelet-derived growth factor receptors A and B (PDGFRA and PDGFRB). Accumulating evidence suggests an interplay between RTK signaling and autophagy [64]. In particular, EGFR activation has been shown to inhibit autophagy via Beclin-1 phosphorylation [65]; KDR activation suppresses autophagy by up-regulating FoxO1 expression; and ABL1 regulates early and late stages of autophagy by phosphorylating Beclin-1 and promoting lysosomal trafficking, respectively [66]. Furthermore, mutant KIT in acute myeloid leukemia (AML) cells triggers autophagy via STAT3 activation. Inhibition of PDGFRs can induce autophagy through PI3K-AKT pathway [67].

This detailed analysis points to several targets whose pro- or anti-autophagic roles are consistent with experimental data. It also shows that the ATG proteins, except for mTOR, are not necessarily the direct targets of autophagy modulators; and a broad number of activators, inhibitors and dualmodulators target instead proteins involved in the transcription regulation of genes involved in autophagy, or in intracellular signal transduction via protein kinases and $\mathrm{Ca}^{2+}$ and $\mathrm{Na}^{+}$channels, mainly. Notably, six targets interact with all three modulators (see the Venn diagram in Fig 1 (right)): two nuclear hormone receptors (NR1I2 and ESR1) two proteins involved in potassium channeling and transport (KCNH2 and ATP4A, respectively), a GPCR (ADORA2A), and the casein kinase II subunit $\alpha$ (CSNK2A1, also known as CK2 $\alpha$ ) (highlighted in yellow in Supplementary Table S2).

\subsection{Autophagy Modulation Targets Are Highly Expressed in The Liver and in The Brain}

Tissue-based enrichment analysis using the ARCHS 4 database [68] suggests that autophagy modulation targets are highly expressed in the liver and in the brain (including various brain regions: cingulate gyrus, superior frontal gyrus, prefrontal cortex, dorsal striatum, and midbrain) (Figure 3c). This could be attributed to the occurrence of aggregation-prone diseases in both organs, e.g. ATZ aggregation in the liver, and many neurodegenerative disorders in the brain. Compared to other tissues, 165 targets were found to have elevated expression levels in the brain using the Human Protein Atlas [69] (Supplementary Table S2).

Overall, our results in the above two subsections show that target proteins of autophagy modulators are commonly expressed in the brain and liver issues and mainly function as tyrosine kinases, calcium channels, aminergic GPCRs, or nuclear hormone receptors. Few frequent targets directly take part in the canonical autophagy pathway, but many of them regulate the expression and activity of core ATG proteins through transcriptional regulation and signaling pathways. We next carried out an in-depth analysis of these pathways toward understanding the pharmacological mechanisms that underlies autophagy modulation.

\subsection{Functional Analysis of The Targets Reveals Enriched Pathways Implicated in Autophagy Modulation}

Figure 4a displays the Gene Ontology (GO) annotation terms enriched in targets of autophagy modulators. Top 20 GO Cellular Components includes nucleus, extrinsic components of cytoplasmic side of plasma membrane, and voltage-gated calcium/sodium channel complex. Top 20 GO Molecular Functions includes various protein kinase activity- and ion channel-related terms. Top 20 Biological Processes comprise a variety of protein phosphorylation and signal transduction-related processes (see Supplementary Table S5 for more extended lists). These results are consistent with our observations in Section 2.3 and highlight the importance of RTK signaling, $\mathrm{Ca}^{2+}$ signaling, and downstream gene transcription regulation. To quantitatively assess the major pathways affected by autophagy modulators, we carried out pathway enrichment analysis using the KEGG database [70]. 
bioRxiv preprint doi: https://doi.org/10.1101/2020.03.25.007344; this version posted March 25, 2020. The copyright holder for this preprint (which was not certified by peer review) is the author/funder, who has granted bioRxiv a license to display the preprint in perpetuity. It is made available under aCC-BY 4.0 International license.

10 of 26
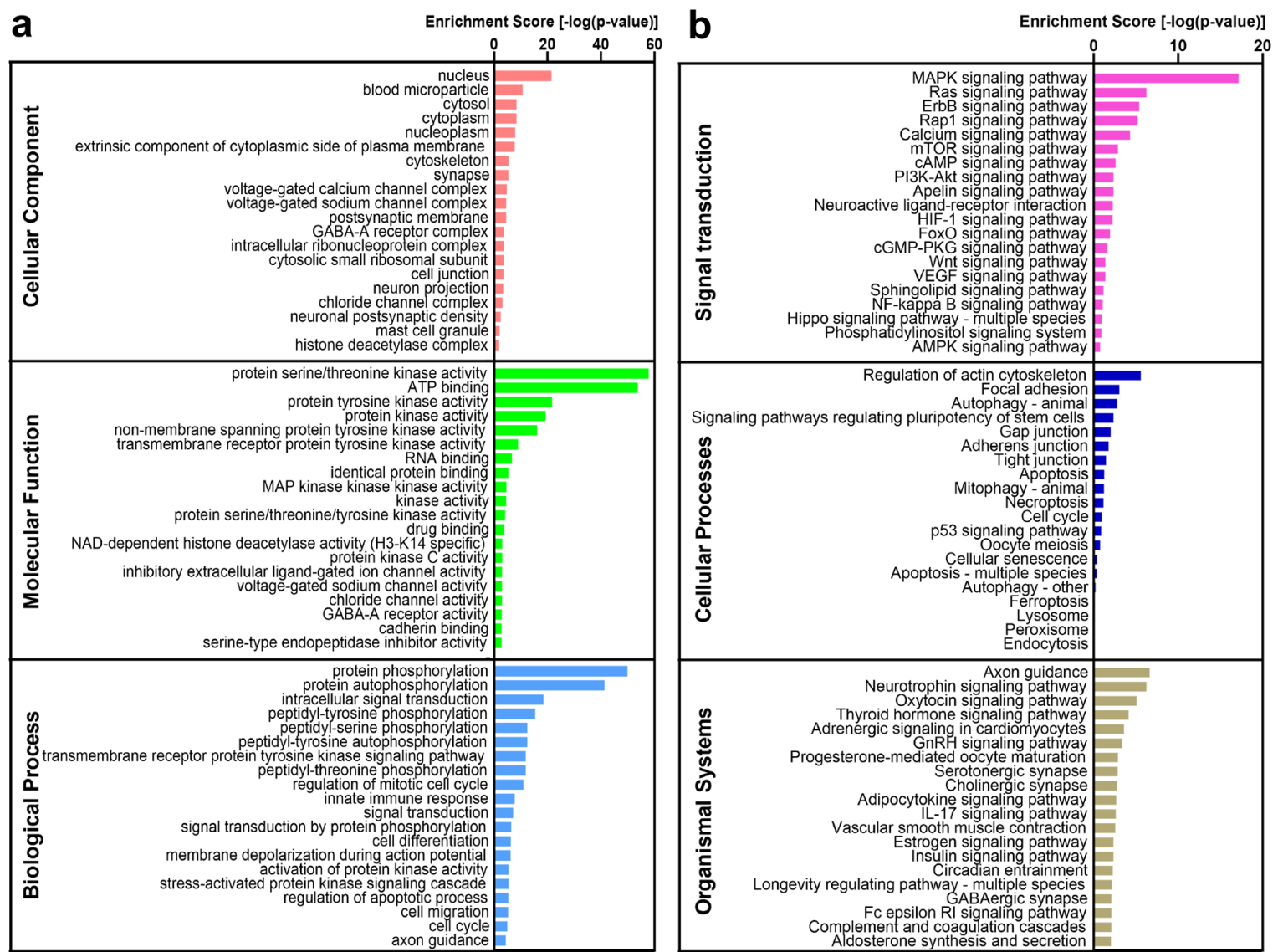

C

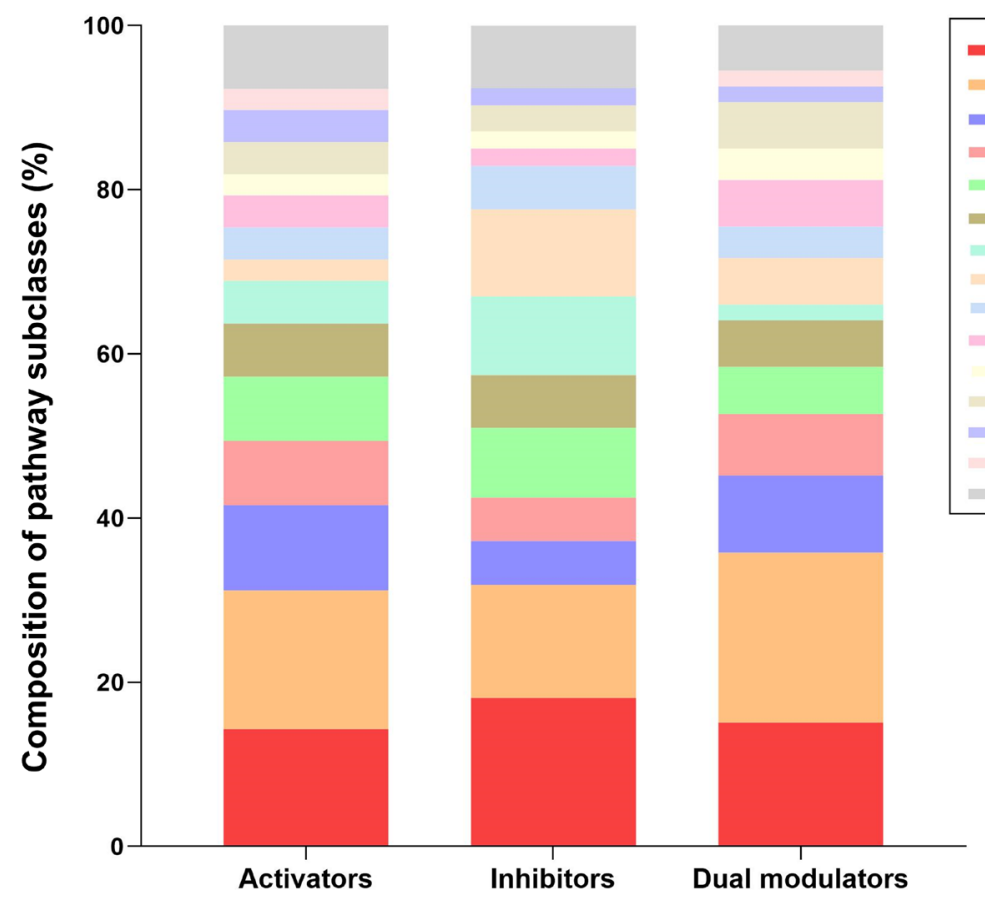

\begin{tabular}{|l|}
\hline Signal transduction \\
Endocrine system \\
Neural diseases \\
Nervous system \\
Infectious diseases \\
Cellular processes \\
Immune system \\
Cancers: specific types \\
Cancers: overview \\
Cardiovascular diseases \\
Circulatory system \\
Metabolic diseases \\
Digestive system \\
Metabolism \\
Others \\
\hline
\end{tabular}

Figure 4. GO annotation and pathway enrichment analysis results based on the targets of autophagy modulators. (a) Enriched GO annotation terms; (b) Enriched KEGG pathways; (c) most common pathways in which autophagy targets take part. The distribution of pathway subclasses targeted by autophagy modulators are shown for each category of autophagy modulators. Note the dominance of signal transduction (red) and endocrine (orange) systems in all categories, the important association of inhibitors with cancers and to some extent, infectious diseases, and the preferential use of activators and dual modulators in treating neural diseases. 
Our analysis indicated that the known and predicted targets of autophagy modulators take part in 294 pathways (Supplementary Table S6). Figure $4 \mathbf{b}$ highlights the top 20 enriched pathways in three distinct KEGG groups: Signal Transduction, Cellular Processes, and Organismal Systems. The enriched pathways in other groups (Human Diseases, and Metabolism, and Genetic Information Processing) can be found in Supplementary Table S6. We found that the Signal Transduction group contains three tiers of autophagy regulation: (1) pathways that directly involve core ATG proteins: mTOR-, PI3K-AKT-, and AMPK-pathways; (2) upstream signaling pathways that regulate the activity of ATG proteins: $\mathrm{Ca}^{2+-}$, cAMP-, and RTK/Rap1/Ras/MAPK-pathways; and (3) transcription pathways that regulate the expression of ATG proteins, mainly HIF-1-, NF- $\mathrm{kB}-$, and FoxO-pathways and their regulators such as nuclear hormone receptors NR1I2 and ESR1.

Though the enrichment scores of pathways in Cellular Processes group are relatively low compared to those in the Signal Transduction group (due to the larger number of proteins involved in Cellular Processes pathways), three autophagy pathways in KEGG were captured by our analysis, including autophagy-animal, autophagy-others, and mitophagy. Various programmed cell death pathways also exhibit relatively high scores, including apoptosis, necroptosis, and ferroptosis. This is due to a complex crosstalk between autophagy and programmed cell death pathways [71-73], which has been shown to process cellular stress signals and confer cell-fate decision [11]. Interestingly, five cell adhesion pathways are top-ranked among cellular processes: regulation of actin cytoskeleton, focal adhesion, gap junction, adherens junction, and tight junction. These pathways regulate the cytoskeletal dynamics, which plays a vital role in the biogenesis of autophagosomes from the ER membrane as well as their translocation and fusion with the lysosomes [74]. Other enriched pathways in Organismal Systems class relate to the physiological functions of autophagy, such as: neuron survival/functioning [75], insulin production/sensitivity [76], circadian rhythm [77], and longevity [78].

We further analyzed the distribution of pathways for each category of autophagy modulators using QuartataWeb [28]. Supplementary Table S6 presents the list of pathways with high enrichment scores ( $p$-value $<0.05$ ), including 63 targeted by activators, 24 targeted by inhibitors, and 106 targeted by dual-modulators. These pathways were further classified into finer subclasses of KEGG pathways. The subclasses of pathways enriched in targets of autophagy activators, inhibitors, and dual-modulators are shown in Figure 4c. Signal Transduction pathways dominate all three categories, along with the endocrine system, a subclass of Organismal Systems. Among the topranked endocrine system pathways, we distinguish the estrogen signaling pathway targeted by 131 activators, 25 inhibitors, and 14 dual-modulators; whereas melanogenesis is exclusively targeted by autophagy activators (see Supplementary Table S6). Recent studies show that rottlerin, an autophagy activator, regulates melanogenesis by targeting the CAMP-CREB signaling pathway [79].

Cancer pathways are also commonly targeted by autophagy modulators (Figure 4c). This is particularly clear in the case of inhibitors. Our results reflect the fact that many autophagy modulators are anti-cancer drugs, which trigger programed cell death and introduce cellular stresses. In response to such stresses, cancer cells may trigger autophagy at the same time to protect themselves. Autophagy is a first rescue mechanism for cells before proceeding to cell death in the presence of stronger insults [11], and its interference may thus delay, if not prevent, tumor cell death. Thus, autophagy inhibition has been identified as a strategy of cancer therapeutics [80]. In particular, breast cancer pathway is highly enriched in targets of autophagy inhibitors ( $p$-value $=1.95 \mathrm{e}-5$ ) but not activators or dual-modulators. This is consistent with the observation that autophagy inhibitors such as SB02024 increase the sensitivity of breast cancer cells to chemotherapy [81].

We also noticed that autophagy inhibitors target more immune system pathways and infection diseases than activators and dual-modulators (respective green and cyan portions of the bars in Figure 4c). Immune pathways specific to autophagy inhibition include: Fc receptor (FCR) signaling, Toll-like receptor (TLR) signaling, T cell receptor (TCR) signaling, c-type lectin receptor (CLR) signaling and chemokine signaling pathways (Supplementary Table S6). It has been reported that stimulation of TLRs, FcRs, TCRs, and CLRs can induce autophagy $[82,83]$. We thus hypothesize that the mechanism of action of selected autophagy inhibitors is through blockage of these pathways. 
Taken together, our enrichment analysis reveals that autophagy activators, inhibitors, and dualmodulators usually affect the same group of pathways, although a few selected pathways seem to be specifically targeted by autophagy activators (e.g. melanogenesis pathway) or inhibitors (e.g. TLR pathway). Signal transduction mechanism shared by the three categories of autophagy modulators include those regulating the activity of ATG proteins directly (e.g. AKT pathway) or indirectly (e.g. calcium pathway), in addition to those involved in their transcriptional regulation (e.g. NF- $\kappa B$ and NHR pathways). We next investigated how different signal transduction pathways interconnect to control/regulate autophagy.

\subsection{PKA, PI3K, AKT, and mTOR Play a Central Signal Transduction Role in Mediating Autophagy}

We identified seven signal transduction pathways highly enriched in targets of all three categories of autophagy modulators: cAMP-, cGMP-PKG-, calcium-, Rap1, Ras, MAPK-, and PI3KAKT-signaling pathways, schematically shown in Figure 5a. These pathways contained each a large number (between 43 and 108) of targets of autophagy modulators, and they were each targeted by a large number (39 to 73) modulators (see the rows colored light blue in Supplementary Table S6).
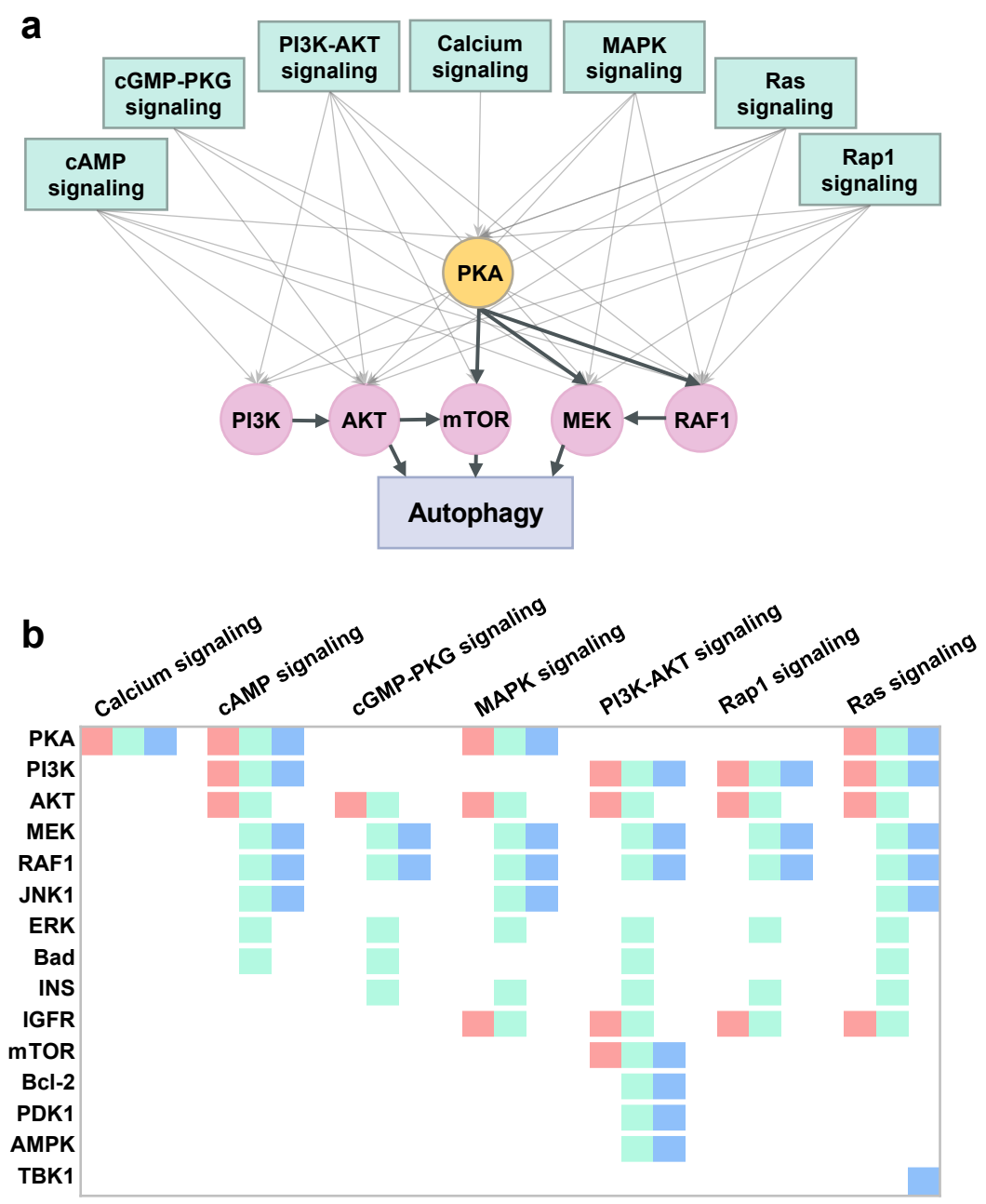

Figure 5. Signal transduction pathways most frequently affected by autophagy modulators and the most promiscuous targets belonging to these pathways. (a) Schematic diagram of the seven ATGsignal transduction pathways and highly promiscuous targets participating in those pathways (indicated by the gray arrow pointing from the pathway to the protein). The target proteins are shown in light violet circles, except for a pivotal target (PI3K) (shown in yellow). Bold face black arrow from $\mathrm{X}$ to $\mathrm{Y}$ means that $\mathrm{X}$ regulates $\mathrm{Y}$. (b) Target-pathway mapping for the most prominent ATG-signal transduction pathways (abscissa) and ATG-signaling targets (ordinate). The color blocks associated with each protein indicate that it is targeted by autophagy activators (green), inhibitors (red), and dualmodulators (blue). Note that mTOR is also targeted by all three groups of modulators. 
Next, we analyzed the known and predicted targets of autophagy modulators and identified those shared by one or more of these pathways as well as the canonical autophagy pathway from KEGG. This led to 15 ATG-signaling targets. These target proteins distinguished by their enhanced propensity to be involved in ATG signal transduction are PKA, PI3K, AKT, MEK1/2, Raf1, JNK1, ERK, Bad, INS, IGFR, mTOR, PP2A, Bcl-2, PDK1, AMPK, and TBK1. Among them PKA and PI3K take part each in four of these seven signal transduction pathways (Figure $5 \mathbf{b}$ ), and AKT in six pathways, and as such they play a key role in mediating autophagic responses. A detailed mapping of these targets to signal transduction pathways is presented in Figure $5 \mathbf{b}$. Three color-coded entries therein indicate whether the ATG-signaling target is associated with an autophagy activator (green), inhibitor (red), and/or dual-modulator (blue). We note in Figure $\mathbf{5 b}$ (and in Supplementary Table S2) that PKA, PI3K and mTOR are targeted by all three categories of autophagy modulators, further supporting the key role of PKA and PI3K, and highlighting that of mTOR as well. The diagram also shows that AKT, MEK1/2, Raf1, and ERK take part in at least five pathways. Interestingly, the MAPK pathway components MEK1/2, Raf1 and JNK1 are targeted by both activators and dual-modulators but not inhibitors. In contrast AKT and IGFR are targeted by activators and inhibitors, but not dualmodulators.

Overall, this analysis draws attention to the central role of PKA as a mediator of ATG signal transduction events. PKA regulates three key players, mTOR, MEK and Raf1, which in turn regulate autophagy (Figure 5a). We also note the critical role of PI3K that regulates AKT, which in turn regulates mTOR. We construct next a comprehensive model of ATG signal transduction network which provides an integrated view of these complex interactions.

\subsection{An Integrative Roadmap for Autophagy Modulation}

We constructed a unified signaling network that integrates the above described pathways, which permits us to visualize how targets of autophagy modulators regulate autophagy (see Figure 6a). Core ATG proteins are colored yellow; the associated transcription factors are in light blue. The above described major ATG-signal transduction pathways are displayed in distinct colors as described in the caption, and the crosstalk between these pathways are schematically shown in Figure 6b. We also indicated in gray boxes the six proteins that are targeted by all three categories of autophagy modulators. This comprehensive network of ATG-signal transduction pathways further highlights the role of PKA (at the center) as a key mediator of the effects of autophagy modulators, bridging various signal transduction events to autophagy and regulating the activity and gene expression of ATG proteins. Yellow ellipses highlight key ATG-signal transduction proteins.

This model provides a comprehensive framework for quantitative analysis of ATG-signal transduction pathways and opens the way to building polypharmacological treatment hypotheses. Figure 6a indicates several autophagy modulators that targets different components, including those binding to upstream channels/receptors such as calcium or potassium channels (top left), or the GPCR ADORA2A (top right) and the downstream key signal transduction elements such as PI3K, AKT, mTORC1, or transcription factors such as HIF-1 $\alpha$. 
bioRxiv preprint doi: https://doi.org/10.1101/2020.03.25.007344; this version posted March 25, 2020. The copyright holder for this preprint (which was not certified by peer review) is the author/funder, who has granted bioRxiv a license to display the preprint in perpetuity. It is made available under aCC-BY 4.0 International license.

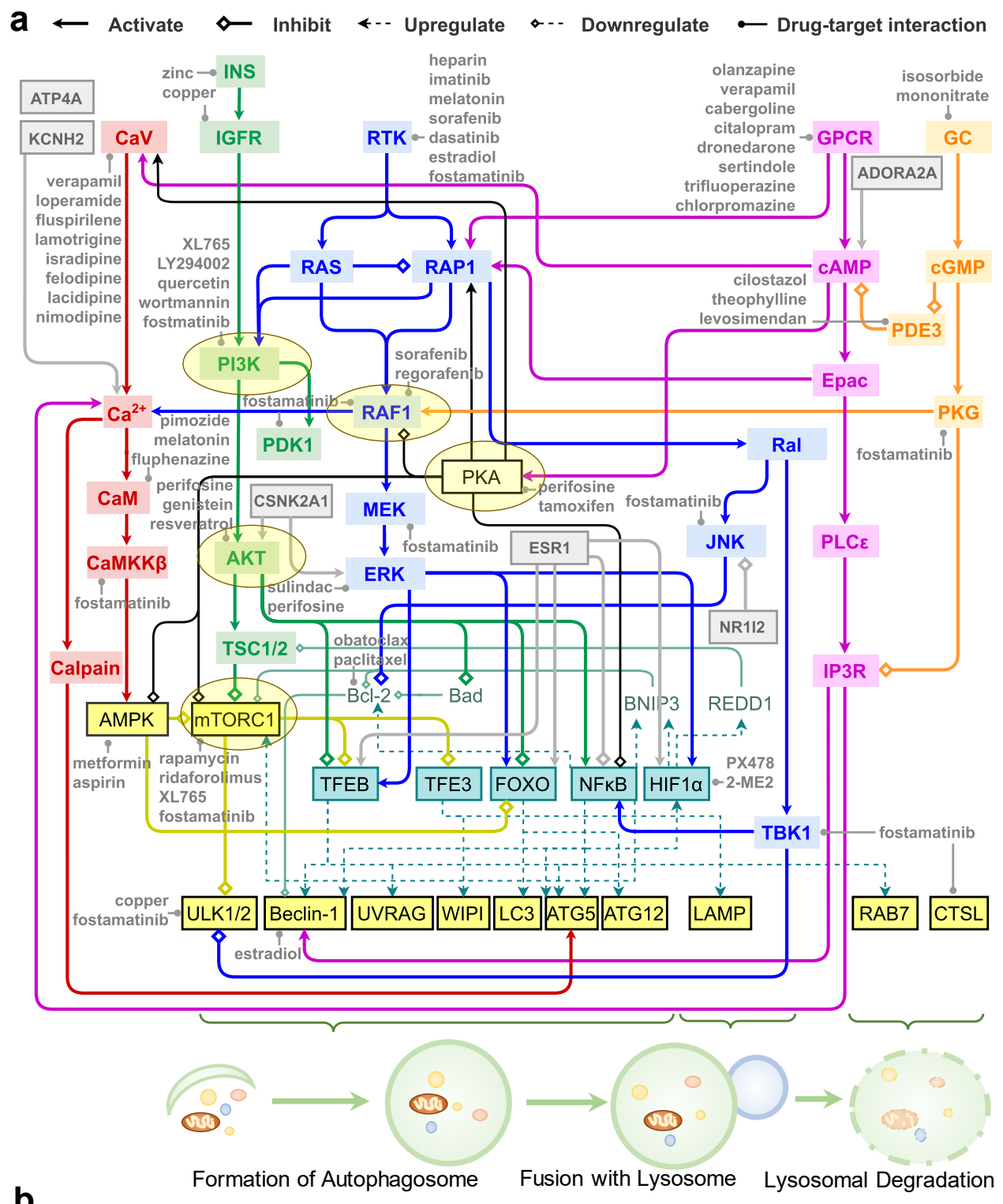

b

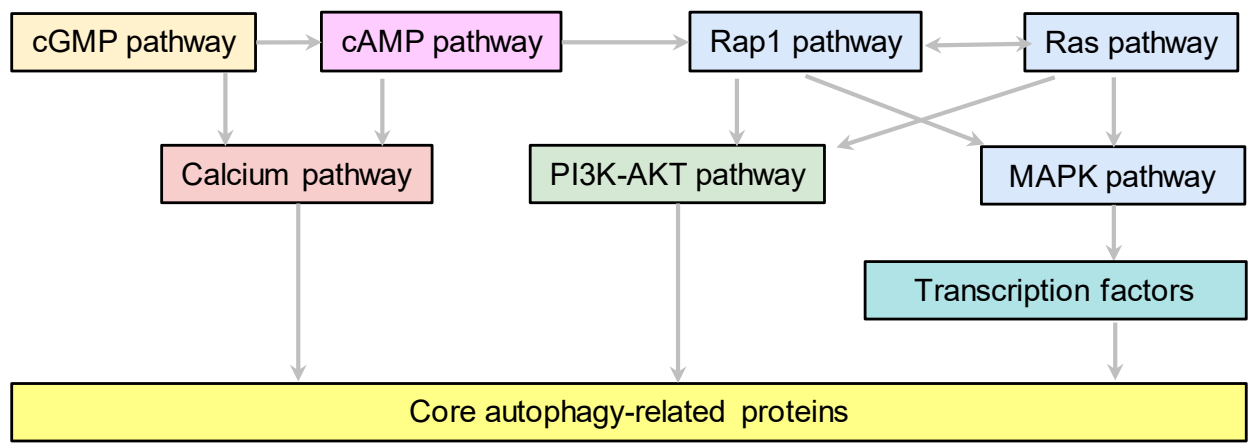

Figure 6. A network of pathways connecting intracellular signal transduction events to autophagy modulation. (a) A unified signaling network mediate the effects of autophagy modulators on signaling transduction; (b) The regulatory dependency of signaling transduction modules. Color code: calcium signaling (red), cAMP signaling (violet), cGMP-PKG signaling (orange), Rap1/Ras/MAPK signaling (blue), PI3K-AKT signaling (green), core autophagy proteins (yellow), and transcription factors (cyan). The core autophagy proteins were grouped (indicated by braces) by their roles in regulating three phases of autophagy: formation of autophagosome, fusion with lysosome, and lysosomal degradation. The key ATG-signal transduction proteins shown in Figure 5a (except MEK) are highlighted by the yellow ellipses. Selected modulators of key targets are listed in gray fonts. See the code for different types of actions indicated by arrow ends on top. 


\section{Discussion}

Pharmacological modulation of autophagy has great therapeutic potential and attracted a great deal of attention in the past two decades [18]. A variety of autophagy-focused databases have been developed to provide annotation information for autophagy-related proteins (such as Autophagy Database [84]), the interactions of autophagy components (e.g., Autophagy Regulatory Network [85], THANATOS [86]), and autophagy-modulating small molecules (e.g., AutophagySMDB [87], HAMdb [88]). These resources contain extensive data on autophagy that enables systems-level analyses of its regulation. In the present study, we mined these databases, manually curated the data in the light of recent literature, and identified a structurally and functionally diverse set of 225 drugs that are known to activate, inhibit, or modulate, autophagy supported by direct experimental evidence.

The experimentally verified (known) targets of these drugs are available in databases such as DrugBank [25]. However, it is still far from a complete understanding of their actions and side effects. QSP methods/tools have been developed in this endeavor, which aim to utilize existing knowledge on networks of interactions among drugs, proteins, and genes for discover new drug targets, reveal underlying mechanisms, and identify repurposed drugs [89,90]. For example, matrix factorizationbased machine learning algorithms have applied to the prediction of novel drug-target interactions $[26,27,91]$. Pathway/network analysis tools have been developed to study drug effects [28,92-94].

In this study, our QSP analysis led to the identification of 1,837 experimentally verified (known) and 368 computationally predicted (new) interactions between these autophagy modulators and their 993 known and 12 new target proteins, involved in 294 pathways. Many new interactions predicted using our machine learning method through QuartataWeb interface [28] were found to be consistent with recent experiments. For example, an interaction between fluphenazine and dopamine receptor D3 which was not reported in DrugBank (and therefore not included in our input dataset) was predicted with high confidence. Notably, this drug was recently reported to have a sub-nanomolar binding affinity on DRD3 $\left(K_{i}=0.11 \mathrm{nM}\right)$ [50]. Among targets we predicted, the translocator protein (TSPO) has been reported to inhibit mitophagy downstream of the PTEN-induced kinase 1 (PINK1)Parkin pathway [95]. Overall, we found experimental evidence in support of 75 (out of 368) predicted pairs, strongly suggesting that the other predictions could as well indicate possible interactions (either repurposable actions or side effects) of existing drugs. Such machine-learning based predictions may thus help generate novel hypotheses worth further testing, or interpret experimental observations. As an example, we predict that olanzapine may interact with calmodulin, implying that the reported olanzapine-induced AMPK activation may be partially mediated by the calmodulinCaMKK $\beta$-AMPK pathway [42,43].

The dominance of the kinase family members among targets of autophagy modulators directed us to focus more closely on interconnected signal transduction pathways implicated in autophagy modulation (Figure 5). The dominant role of kinases is consistent with a study of human kinasesubstrate post-translational modification network where phosphorylation plays a key role in regulating autophagy [86]. In addition to the kinases highlighted in Figure 5, as illustrated in Figures 6, core autophagy proteins such as ULK1/2, and AMPK are all kinases. MAPKs and PKA crosstalk to other pathways and thus their roles in regulating autophagy depend on the cellular context. Since kinase pathways are activated in several cancers, differential regulation of autophagy by targeting multiple kinase pathways has been suggested for the development of better cancer therapeutics [96].

Another important class of proteins broadly used as drug targets, GPCRs, are also targeted for autophagy modulations. In this case, GPCRs regulate second messengers $\mathrm{Ca}^{2+}$ and $\mathrm{CAMP}$, and downstream effectors MAPKs, which initiate the major upstream signal transduction that leads to autophagy. It has been suggested that the regulation of autophagy by GPCRs is essential to mediating the effects of hormones/neurotransmitters secreted in response to systemic nutrient fluctuations [58]. Many GPCRs (e.g. ADA1s) and calcium channels (e.g. CAC1s) are frequent targets showing that the corresponding autophagy modulators mediate by upstream signaling pathways, rather than the canonical mTOR pathway.

Turning to the NHRs, our analysis highlighted two important transcription factors directedly targeted by autophagy modulators: HIF-1 $\alpha$ (targeted by PX-478 and 2-methoxyestradiol) and NF-kB 
(targeted by glucosamine and acetylsalicylic acid). Notably both of these, and other transcription factors such as FoxO and TFEB are regulated by estrogen receptor/NHR ESR1, hence the frequent observation of ESR1 as a major target. Another NHR distinguished by its high promiscuity was NR1I2, which directly regulates the kinase pathways.

The key autophagy proteins that these transcription factors and/or NHRs regulate (e.g. Beclin1, LC3, ATG5) are illustrated in Figure 6a. Though transcription factors such as TFEB, TFE3, and FoxO are not directly targeted by autophagy modulators, their regulators such as AKT, mTOR, and AMPK appear to be targets. The presence of FoxO, HIF-1-, and NF- $\kappa \mathrm{B}$ signaling pathways among the top 20 enriched pathways (Figure 4) further confirmed that the regulation of autophagic gene expression is an important mechanism exploited by many autophagy modulators.

Note that Figure 6a only illustrated representative crosstalks between the pathways mentioned above. The signaling network regulating autophagy may be rewired by specific crosstalks in different cell types. For example, in KRAS-driven cancers, calmodulin binds KRAS, and can directly activate PI3K and AKT [97,98]. Further, our classification of autophagy modulators as activators, inhibitors, and dual-modulators is limited by the existing experimental evidence. Though many targets only interact with either activators or inhibitors (Supplementary Figure S2), given the complex pathway crosstalk shown in Figure 6a, it is possible that current autophagy activators and inhibitors are in fact dual-modulators. For example, the most promiscuous autophagy inhibitor fostamatinib is known to inhibit serine/threonine-protein kinase tousled-like 2 (TLK2), a negative regulator of amino acid starvation-induced autophagy [99]. Thus, fostamatinib may enhance autophagy via TLK2, although there is no experimental data in support of this, yet. Furthermore, Rev-Erb agonists SR-9009 and SR9011 inhibit autophagy through nuclear receptor subfamily 1 group D members 1 and 2 (NR1D1/2) [100]. Since NR1D1 mediates the regulation of autophagy rhythms by circadian clock [100], whether the inhibition of NR1D1 leads to the enhancement or suppression of autophagy might depend on the timing and cellular state. Melanogenesis and TLR signaling pathways are exclusively targeted by autophagy activators or inhibitors, respectively. Whether it implies specific mechanisms underlying autophagy activation and inhibition requires further investigations.

PKA, downstream of cAMP signaling, takes part in all three tiers of autophagy regulation: (i) it inhibits core autophagy proteins AMPK and mTOR; (ii) it regulates upstream MAPK signaling; and (iii) it inhibits NF- $\kappa B$ signaling. The drugs targeting PKA involve autophagy activators (e.g. fasudil), inhibitors (e.g. fostamatinib), and dual-modulators (e.g. metformin). PKA is key regulator that governs various cellular processes. Interestingly, a recent high-throughput screening study in yeast suggested that many substrates or downstream autophagy proteins (e.g. ATG10, ATG14, ATG15, and ATG16) regulated by PKA turn are themselves PKA regulators as well [101]. This implies the presence of multiple positive and negative feedback loops coupling PKA to autophagic responses. It has been reported that mammalian PKA activates mTORC1 while, in yeast, TORC1 can indirectly activate PKA through a homolog of a ribosomal protein S6 kinase B1 (RPS6KB1) [102]. All these observations point to the utility of a thorough study of the feedback regulation between PKA and ATG proteins in human cells. In this context, dynamical modeling approaches promise to offer helpful pointers [11,103-106].

As shown in Figure 4, targets of autophagy modulators are involved in neural diseases, infectious diseases, cancers, cardiovascular diseases, and metabolic diseases pathways; they are upregulated in the liver and brain tissues (Figure 3c). This observation may be partially due to the presence of antipsychotic drugs (e.g. olanzapine and pimozide) and liver disease drugs (e.g. carbamazepine and resveratrol) in our input list of autophagy modulators, both developed for eliminating toxic aggregates. However, it also reflects the crucial role of autophagy in liver and brain health and diseases [107-109] and justifies the attempts of repurposing autophagy modulators against liver and brain diseases [21-23]. The targets involved in cardiovascular diseases are mainly calcium channels. Intracellular $\mathrm{Ca}^{2+}$ can both up- and down-regulate autophagy and plays a key role in the crosstalk between autophagy and apoptosis [11]. The interplay between autophagy and apoptosis renders opposing roles of autophagy as a therapeutic target for cardiovascular diseases. For example, in ischemic heart disease, enhanced autophagy is desired for cardioprotective effects via ROS 
reduction; however, alleviating chemotherapy-induced cardiotoxicity requires suppression of autophagy [110].

Our enrichment analysis also show that many targets of autophagy modulators are involved in apoptosis (e.g. p53, Bcl-2, and caspase 8), necroptosis (e.g. caspase 8, RIPK1, and TNF), and ferroptosis (e.g. p53, 15LOX, and ACSL4) pathways (Figure 4 and Supplementary Figure S6). In recent work, we have demonstrated the pivot roles of these targets in regulating distinct cell death pathways [103,111-113]. We also found that due to complex crosstalks among pathways, targeting autophagy, necroptosis, and ferroptosis using polypharmacological strategies can lead to better therapeutic effects in terms of cell survival $[114,115]$. The present study shows that the key regulators of cell death are targeted by autophagy modulators, in accord with the crosstalk between autophagy and these cell death pathways. The integrated network constructed based on our analysis (Figure 6) may serve as an excellent framework for pursuing quantitative analyses shedding light to the biological and biomedical implications of such crosstalks and opening new directions for designing polypharmacological strategies for monitoring or altering cell survival/death decisions.

\section{Materials and Methods}

\subsection{Data Collection}

We selected 225 drugs from DrugBank v5.1.1, which have at least one target and are also known as autophagy modulators (Supplementary Table S1). We classify the selected modulators into three groups: activators, inhibitors, and dual-modulators by manually searching for direct experimental evidence in support of their specific role in the literature. A dataset of 993 known targets were retrieved from DrugBank (Supplementary Table S2).

\subsection{Drug-Target Interaction Prediction}

New drug-target interactions were predicted using our PMF-based machine learning algorithm [26-28]. We represented the known interactions between $N$ drugs and $M$ targets as a sparse matrix $\boldsymbol{R}$. We then decomposed $\boldsymbol{R}$ into a drug matrix $\boldsymbol{U}$ and a target matrix $\boldsymbol{V}$, by learning the optimal $D$ latent variables to represent each drug and each target using the PMF algorithm. The product of $\boldsymbol{U}^{T}$ and $\boldsymbol{V}$ reconstructed $\boldsymbol{R}$ by assigning each of the unknown entries in $\boldsymbol{R}$ a value, which represents the confidence score for a novel drug-target interaction prediction:

$$
\boldsymbol{R}_{N \times M}=\boldsymbol{U}_{N \times D}^{T} \boldsymbol{V}_{D \times M}
$$

Using this method, we trained a PMF model based on 14,983 drug-target interactions between 5,494 drugs and 2,807 targets from DrugBank 5.1.1. We evaluated the confidence scores in the range [0, 1] for each predicted drug-target interaction. We selected the interactions with confidence scores higher than 0.6 within the top 40 predicted targets for each input drug. This led to 368 novel interactions (Supplementary Table S4).

\subsection{Drug-Drug Similarity Analysis}

We evaluated the structural similarity between each pair of autophagy modulators by calculating the Tanimoto distance between their 2D structure fingerprints using the RDKit software [116]. We evaluated the functional similarity of two drugs based on their interactions pattern with known targets. Specifically, we represent each drug $i$ by a vector $\boldsymbol{d}_{i}$ of values of 0 or 1 (depending on the absence or existence of an interaction between this drug and the corresponding target) in the matrix R. Interaction-pattern similarities between drugs $i$ and $j$ are then evaluated by calculating the cosine distance between their vectors $\boldsymbol{d}_{i}$ and $\boldsymbol{d}_{j}$ :

$$
1-\left(\boldsymbol{d}_{i} \cdot \boldsymbol{d}_{j}\right) /\left(\left|\boldsymbol{d}_{i}\right|\left|\boldsymbol{d}_{j}\right|\right)
$$

\subsection{Pathway Enrichment Analysis}


We enriched the KEGG pathways (version March 2018, homo sapiens) [117] by the 225 drugs with 993 known and 12 predicted targets. 294 pathways were identified (see Supplementary Table S6). We calculated the hypergeometric $p$-values to rank enriched pathways. Our enrichment scores are defined as follows. Given a list of targets, the enrichment $p$-value for pathway $i\left(P_{i}\right)$ is the probability of randomly drawing $k_{0}$ or more targets that belong to $P_{i}$ :

$$
P_{i}=\sum_{k_{0} \leq k \leq m} \frac{\left(\begin{array}{l}
K \\
k
\end{array}\right)\left(\begin{array}{l}
M-K \\
m-k
\end{array}\right)}{\left(\begin{array}{l}
M \\
m
\end{array}\right)}
$$

where $m$ is the total number of targets we identified, $M$ is the total number of human pathways in KEGG, $K$ is the number of targets that take part in the examined pathway $i$, and $k_{0}$ is the number of targets we identified that are involved in pathway $i$. In order to account for multiple testing, we use the Benjamini-Hochberg method [118] to adjust the calculated $p$-values by introducing a False Discovery Rate (FDR) correction. The FDR is the fraction of false significant pathways maximally expected from the significant pathways we identified, which is bounded by the cutoff of the adjusted $p$-values. Specifically, the adjusted $p$-value, $p_{i}^{*}$, for the $i$ th pathway is:

$$
p_{i}^{*}=\min _{k=i \ldots m}\left\{\min \left(\frac{p_{k} m}{i}, 1\right)\right.
$$

where $m$ is the total number of pathways. Supplementary Table S6 lists these $p$-values for pathway enrichments based on both known and predicted targets.

Supplementary Materials: Supplementary materials can be found at www.mdpi.com/xxx/s1. The source code and supplementary data are available at http://github.com/liubing1020/autophagy.

Author Contributions: Conceptualization, Q.S., F.P., B.L, and I.B.; methodology, Q.S. and F.P.; investigation, Q.S., F.P., B.L. and I.B.; data curation, Q.S.; writing-original draft preparation, Q.S. and B.L; writing-review and editing, F.P. and I.B.; visualization, Q.S. and B.L.; supervision, B.L. and I.B.; funding acquisition, I.B. All authors have read, and agreed on, the manuscript.

Funding: This research was funded by National Institutes of Health awards P01DK096990, P30DA035778, and P41GM103712 to I.B.

Acknowledgments: F.P. wishes to thank Dr. D. Lansing Taylor for his mentorship.

Conflicts of Interest: The authors declare no conflict of interest. The funders had no role in the design of the study; in the collection, analyses, or interpretation of data; in the writing of the manuscript, or in the decision to publish the results.

\section{References}

1. Wang, Y.; Xu, W.; Yan, Z.; Zhao, W.; Mi, J.; Li, J.; Yan, H. Metformin induces autophagy and G0/G1 phase cell cycle arrest in myeloma by targeting the AMPK/mTORC1 and mTORC2 pathways. J Exp Clin Cancer Res 2018, 37, 63, doi:10.1186/s13046-018-0731-5.

2. Levine, B.; Kroemer, G. Biological functions of autophagy genes: a disease perspective. Cell 2019, 176, 11-42, doi:10.1016/j.cell.2018.09.048.

3. Rabinowitz, J.D.; White, E. Autophagy and metabolism. Science 2010, 330, 1344-1348, doi:10.1126/science.1193497.

4. Mizushima, N. Autophagy: process and function. Genes Dev 2007, 21, 2861-2873, doi:10.1101/gad.1599207.

5. Levine, B. Cell biology: autophagy and cancer. Nature 2007, 446, 745-747, doi:10.1038/446745a. 
6. Lorin, S.; Hamai, A.; Mehrpour, M.; Codogno, P. Autophagy regulation and its role in cancer. Semin Cancer Biol 2013, 23, 361-379, doi:10.1016/j.semcancer.2013.06.007.

7. Mathew, R.; Karantza-Wadsworth, V.; White, E. Role of autophagy in cancer. Nat Rev Cancer 2007, 7, 961-967, doi:10.1038/nrc2254.

8. Kim, Y.C.; Guan, K.L. mTOR: a pharmacologic target for autophagy regulation. J Clin Invest 2015, 125, 25-32, doi:10.1172/JCI73939.

9. Mizushima, N. The role of the Atg1/ULK1 complex in autophagy regulation. Curr Opin Cell Biol 2010, 22, 132-139, doi:10.1016/j.ceb.2009.12.004.

10. Graef, M.; Nunnari, J. A role for mitochondria in autophagy regulation. Autophagy 2011, 7, 1245-1246, doi:10.4161/auto.7.10.16508.

11. Liu, B.; Oltvai, Z.N.; Bayir, H.; Silverman, G.A.; Pak, S.C.; Perlmutter, D.H.; Bahar, I. Quantitative assessment of cell fate decision between autophagy and apoptosis. Sci Rep 2017, 7, 17605.

12. Decuypere, J.P.; Bultynck, G.; Parys, J.B. A dual role for $\mathrm{Ca}(2+)$ in autophagy regulation. Cell Calcium 2011, 50, 242-250, doi:10.1016/j.ceca.2011.04.001.

13. Racioppi, L.; Means, A.R. Calcium/calmodulin-dependent protein kinase kinase 2: roles in signaling and pathophysiology. J Biol Chem 2012, 287, 31658-31665, doi:10.1074/jbc.R112.356485.

14. Yousefi, S.; Perozzo, R.; Schmid, I.; Ziemiecki, A.; Schaffner, T.; Scapozza, L.; Brunner, T.; Simon, H.U. Calpain-mediated cleavage of Atg5 switches autophagy to apoptosis. Nat Cell Biol 2006, 8, 1124-1132, doi:10.1038/ncb1482.

15. Pan, T.; Rawal, P.; Wu, Y.; Xie, W.; Jankovic, J.; Le, W. Rapamycin protects against rotenone-induced apoptosis through autophagy induction. Neuroscience 2009, 164, 541-551, doi:10.1016/j.neuroscience.2009.08.014.

16. Li, Y.; Wang, X.; Wei, Z.; Mao, H.; Gao, M.; Liu, Y.; Ma, Y.; Liu, X.; Guo, C.; Zhang, L., et al. Pretreatment with wortmannin alleviates lipopolysaccharide/d-galactosamine-induced acute liver injury. Biochem Biophys Res Commun 2014, 455, 234-240, doi:10.1016/j.bbrc.2014.10.152.

17. O'Reilly, L.P.; Long, O.S.; Cobanoglu, M.C.; Benson, J.A.; Luke, C.J.; Miedel, M.T.; Hale, P.; Perlmutter, D.H.; Bahar, I.; Silverman, G.A., et al. A genome-wide RNAi screen identifies potential drug targets in a C. elegans model of alpha1-antitrypsin deficiency. Hum Mol Genet 2014, 23, 5123-5132, doi:10.1093/hmg/ddu236.

18. Galluzzi, L.; Bravo-San Pedro, J.M.; Levine, B.; Green, D.R.; Kroemer, G. Pharmacological modulation of autophagy: therapeutic potential and persisting obstacles. Nat Rev Drug Discov 2017, 16, 487-511, doi:10.1038/nrd.2017.22.

19. Towers, C.G.; Thorburn, A. Therapeutic targeting of autophagy. EBioMedicine 2016, 14, 15-23, doi:10.1016/j.ebiom.2016.10.034.

20. Cai, Z.; Yan, L.J. Rapamycin, autophagy, and alzheimer's disease. J Biochem Pharmacol Res 2013, 1, 8490.

21. Hidvegi, T.; Ewing, M.; Hale, P.; Dippold, C.; Beckett, C.; Kemp, C.; Maurice, N.; Mukherjee, A.; Goldbach, C.; Watkins, S., et al. An autophagy-enhancing drug promotes degradation of mutant alpha1-antitrypsin $Z$ and reduces hepatic fibrosis. Science 2010, 329, 229-232, doi:10.1126/science.1190354.

22. Li, J.; Pak, S.C.; O'Reilly, L.P.; Benson, J.A.; Wang, Y.; Hidvegi, T.; Hale, P.; Dippold, C.; Ewing, M.; Silverman, G.A., et al. Fluphenazine reduces proteotoxicity in C. elegans and mammalian models of alpha-1-antitrypsin deficiency. PLoS One 2014, 9, e87260, doi:10.1371/journal.pone.0087260. 
23. Wang, Y.; Cobanoglu, M.C.; Li, J.; Hidvegi, T.; Hale, P.; Ewing, M.; Chu, A.S.; Gong, Z.; Muzumdar, R.; Pak, S.C., et al. An analog of glibenclamide selectively enhances autophagic degradation of misfolded alpha1-antitrypsin Z. PLoS One 2019, 14, e0209748, doi:10.1371/journal.pone.0209748.

24. Cuomo, F.; Altucci, L.; Cobellis, G. Autophagy function and dysfunction: potential drugs as anti-cancer therapy. Cancers (Basel) 2019, 11, doi:10.3390/cancers11101465.

25. Wishart, D.S.; Feunang, Y.D.; Guo, A.C.; Lo, E.J.; Marcu, A.; Grant, J.R.; Sajed, T.; Johnson, D.; Li, C.; Sayeeda, Z., et al. DrugBank 5.0: a major update to the DrugBank database for 2018. Nucleic Acids Res 2018, 46, D1074-D1082, doi:10.1093/nar/gkx1037.

26. Cobanoglu, M.C.; Liu, C.; Hu, F.; Oltvai, Z.N.; Bahar, I. Predicting drug-target interactions using probabilistic matrix factorization. J Chem Inf Model 2013, 53, 3399-3409, doi:10.1021/ci400219z.

27. Cobanoglu, M.C.; Oltvai, Z.N.; Taylor, D.L.; Bahar, I. BalestraWeb: efficient online evaluation of drugtarget interactions. Bioinformatics 2015, 31, 131-133, doi:10.1093/bioinformatics/btu599.

28. Li, H.; Pei, F.; Taylor, D.L.; Bahar, I. QuartataWeb: an integrated chemical-protein interaction prediction and pathway inference server for polyphamalogical and chemogenomics analysis. Bioinformatics 2020, in press.

29. Sagrillo-Fagundes, L.; Bienvenue-Pariseault, J.; Vaillancourt, C. Melatonin: The smart molecule that differentially modulates autophagy in tumor and normal placental cells. PLoS One 2019, 14, e0202458, doi:10.1371/journal.pone.0202458.

30. Brachmann, S.; Fritsch, C.; Maira, S.M.; Garcia-Echeverria, C. PI3K and mTOR inhibitors: a new generation of targeted anticancer agents. Curr Opin Cell Biol 2009, 21, 194-198, doi:10.1016/j.ceb.2008.12.011.

31. Moriya, S.; Che, X.F.; Komatsu, S.; Abe, A.; Kawaguchi, T.; Gotoh, A.; Inazu, M.; Tomoda, A.; Miyazawa, K. Macrolide antibiotics block autophagy flux and sensitize to bortezomib via endoplasmic reticulum stress-mediated CHOP induction in myeloma cells. Int J Oncol 2013, 42, 1541-1550, doi:10.3892/ijo.2013.1870.

32. Wang, D.; Ji, X.; Liu, J.; Li, Z.; Zhang, X. Dopamine receptor subtypes differentially regulate autophagy. Int J Mol Sci 2018, 19, doi:10.3390/ijms19051540.

33. Trejo-Solis, C.; Jimenez-Farfan, D.; Rodriguez-Enriquez, S.; Fernandez-Valverde, F.; Cruz-Salgado, A.; Ruiz-Azuara, L.; Sotelo, J. Copper compound induces autophagy and apoptosis of glioma cells by reactive oxygen species and JNK activation. BMC Cancer 2012, 12, 156, doi:10.1186/1471-2407-12-156.

34. Liuzzi, J.P.; Guo, L.; Yoo, C.; Stewart, T.S. Zinc and autophagy. Biometals 2014, 27, 1087-1096, doi:10.1007/s10534-014-9773-0.

35. Tsang, T.; Posimo, J.M.; Guidiel, A.A.; Cicchini, M.; Feldser, D.M.; Brady, D.C. Copper is an essential regulator of the autohagic kinases ULK1/2 to dirve lung adenocarcinoma. Nat Cell Biol 2020, in press.

36. Olliaro, P.L.; Haynes, R.K.; Meunier, B.; Yuthavong, Y. Possible modes of action of the artemisinin-type compounds. Trends Parasitol 2001, 17, 122-126, doi:10.1016/s1471-4922(00)01838-9.

37. Wang, J.; Zhang, C.J.; Chia, W.N.; Loh, C.C.; Li, Z.; Lee, Y.M.; He, Y.; Yuan, L.X.; Lim, T.K.; Liu, M., et al. Haem-activated promiscuous targeting of artemisinin in Plasmodium falciparum. Nat Commun 2015, 6, 10111, doi:10.1038/ncomms10111.

38. Shi, X.; Wang, L.; Li, X.; Bai, J.; Li, J.; Li, S.; Wang, Z.; Zhou, M. Dihydroartemisinin induces autophagydependent death in human tongue squamous cell carcinoma cells through DNA double-strand breakmediated oxidative stress. Oncotarget 2017, 8, 45981-45993, doi:10.18632/oncotarget.17520. 
39. Konstat-Korzenny, E.; Ascencio-Aragon, J.A.; Niezen-Lugo, S.; Vazquez-Lopez, R. Artemisinin and its synthetic derivatives as a possible therapy for cancer. Med Sci (Basel) 2018, 6, doi:10.3390/medsci6010019.

40. Niture, S.; Gyamfi, M.A.; Kedir, H.; Arthur, E.; Ressom, H.; Deep, G.; Kumar, D. Serotonin induced hepatic steatosis is associated with modulation of autophagy and notch signaling pathway. Cell Commun Signal 2018, 16, 78, doi:10.1186/s12964-018-0282-6.

41. Kim, J.K.; Kim, Y.S.; Lee, H.M.; Jin, H.S.; Neupane, C.; Kim, S.; Lee, S.H.; Min, J.J.; Sasai, M.; Jeong, J.H., et al. GABAergic signaling linked to autophagy enhances host protection against intracellular bacterial infections. Nat Commun 2018, 9, 4184, doi:10.1038/s41467-018-06487-5.

42. Ikegami, M.; Ikeda, H.; Ishikawa, Y.; Ohsawa, M.; Ohashi, T.; Kai, M.; Kamei, A.; Kamei, J. Olanzapine induces glucose intolerance through the activation of AMPK in the mouse hypothalamus. Eur $J$ Pharmacol 2013, 718, 376-382, doi:10.1016/j.ejphar.2013.08.006.

43. Schmidt, R.H.; Jokinen, J.D.; Massey, V.L.; Falkner, K.C.; Shi, X.; Yin, X.; Zhang, X.; Beier, J.I.; Arteel, G.E. Olanzapine activates hepatic mammalian target of rapamycin: new mechanistic insight into metabolic dysregulation with atypical antipsychotic drugs. J Pharmacol Exp Ther 2013, 347, 126-135, doi:10.1124/jpet.113.207621.

44. Vucicevic, L.; Misirkic-Marjanovic, M.; Paunovic, V.; Kravic-Stevovic, T.; Martinovic, T.; Ciric, D.; Maric, N.; Petricevic, S.; Harhaji-Trajkovic, L.; Bumbasirevic, V., et al. Autophagy inhibition uncovers the neurotoxic action of the antipsychotic drug olanzapine. Autophagy 2014, 10, 2362-2378, doi:10.4161/15548627.2014.984270.

45. Shinde, A.; Hardy, S.D.; Kim, D.; Akhand, S.S.; Jolly, M.K.; Wang, W.H.; Anderson, J.C.; Khodadadi, R.B.; Brown, W.S.; George, J.T., et al. Spleen tyrosine kinase-mediated autophagy is required for epithelial-mesenchymal plasticity and metastasis in breast cancer. Cancer Res 2019, 79, 1831-1843, doi:10.1158/0008-5472.CAN-18-2636.

46. Duan, W.; Guo, M.; Yi, L.; Zhang, J.; Bi, Y.; Liu, Y.; Li, Y.; Li, Z.; Ma, Y.; Zhang, G., et al. Deletion of Tbk1 disrupts autophagy and reproduces behavioral and locomotor symptoms of FTD-ALS in mice. Aging (Albany NY) 2019, 11, 2457-2476, doi:10.18632/aging.101936.

47. Singh, P.; Ravanan, P.; Talwar, P. Death associated protein kinase 1 (DAPK1): a regulator of apoptosis and autophagy. Frontiers in molecular neuroscience 2016, 9, 46, doi:10.3389/fnmol.2016.00046.

48. Ber, Y.; Shiloh, R.; Gilad, Y.; Degani, N.; Bialik, S.; Kimchi, A. DAPK2 is a novel regulator of mTORC1 activity and autophagy. Cell Death Differ 2015, 22, 465-475, doi:10.1038/cdd.2014.177.

49. Manzoni, C.; Lewis, P.A. LRRK2 and Autophagy. Adv Neurobiol 2017, 14, 89-105, doi:10.1007/978-3-31949969-7_5.

50. Davis, M.I.; Hunt, J.P.; Herrgard, S.; Ciceri, P.; Wodicka, L.M.; Pallares, G.; Hocker, M.; Treiber, D.K.; Zarrinkar, P.P. Comprehensive analysis of kinase inhibitor selectivity. Nat Biotechnol 2011, 29, 10461051, doi:10.1038/nbt.1990.

51. Li, P.; Snyder, G.L.; Vanover, K.E. Dopamine targeting drugs for the treatment of schizophrenia: past, present and future. Curr Top Med Chem 2016, 16, 3385-3403, doi:10.2174/1568026616666160608084834.

52. Besnard, J.; Ruda, G.F.; Setola, V.; Abecassis, K.; Rodriguiz, R.M.; Huang, X.P.; Norval, S.; Sassano, M.F.; Shin, A.I.; Webster, L.A., et al. Automated design of ligands to polypharmacological profiles. Nature 2012, 492, 215-220, doi:10.1038/nature11691.

53. Pundir, S.; Martin, M.J.; O'Donovan, C. UniProt Protein Knowledgebase. Methods Mol Biol 2017, 1558, 41-55, doi:10.1007/978-1-4939-6783-4_2. 
54. Santos, R.; Ursu, O.; Gaulton, A.; Bento, A.P.; Donadi, R.S.; Bologa, C.G.; Karlsson, A.; Al-Lazikani, B.; Hersey, A.; Oprea, T.I., et al. A comprehensive map of molecular drug targets. Nat Rev Drug Discov 2017, 16, 19-34, doi:10.1038/nrd.2016.230.

55. Xiang, J.; Liu, X.; Ren, J.; Chen, K.; Wang, H.L.; Miao, Y.Y.; Qi, M.M. How does estrogen work on autophagy? Autophagy 2019, 15, 197-211, doi:10.1080/15548627.2018.1520549.

56. Blessing, A.M.; Rajapakshe, K.; Reddy Bollu, L.; Shi, Y.; White, M.A.; Pham, A.H.; Lin, C.; Jonsson, P.; Cortes, C.J.; Cheung, E., et al. Transcriptional regulation of core autophagy and lysosomal genes by the androgen receptor promotes prostate cancer progression. Autophagy 2017, 13, 506-521, doi:10.1080/15548627.2016.1268300.

57. Yan, S.; Yang, X.; Chen, T.; Xi, Z.; Jiang, X. The PPARgamma agonist Troglitazone induces autophagy, apoptosis and necroptosis in bladder cancer cells. Cancer Gene Ther 2014, 21, 188-193, doi:10.1038/cgt.2014.16.

58. Wauson, E.M.; Dbouk, H.A.; Ghosh, A.B.; Cobb, M.H. G protein-coupled receptors and the regulation of autophagy. Trends Endocrinol Metab 2014, 25, 274-282, doi:10.1016/j.tem.2014.03.006.

59. Lizaso, A.; Tan, K.T.; Lee, Y.H. beta-adrenergic receptor-stimulated lipolysis requires the RAB7mediated autolysosomal lipid degradation. Autophagy 2013, 9, 1228-1243, doi:10.4161/auto.24893.

60. Merlin, J.; Evans, B.A.; Csikasz, R.I.; Bengtsson, T.; Summers, R.J.; Hutchinson, D.S. The M3-muscarinic acetylcholine receptor stimulates glucose uptake in L6 skeletal muscle cells by a CaMKK-AMPKdependent mechanism. Cell Signal 2010, 22, 1104-1113, doi:10.1016/j.cellsig.2010.03.004.

61. Yan, Y.; Jiang, W.; Liu, L.; Wang, X.; Ding, C.; Tian, Z.; Zhou, R. Dopamine controls systemic inflammation through inhibition of NLRP3 inflammasome. Cell 2015, 160, 62-73, doi:10.1016/j.cell.2014.11.047.

62. Dolma, S.; Selvadurai, H.J.; Lan, X.; Lee, L.; Kushida, M.; Voisin, V.; Whetstone, H.; So, M.; Aviv, T.; Park, N., et al. Inhibition of dopamine receptor D4 impedes autophagic flux, proliferation, and survival of glioblastoma stem cells. Cancer Cell 2016, 29, 859-873, doi:10.1016/j.ccell.2016.05.002.

63. Soll, C.; Jang, J.H.; Riener, M.O.; Moritz, W.; Wild, P.J.; Graf, R.; Clavien, P.A. Serotonin promotes tumor growth in human hepatocellular cancer. Hepatology 2010, 51, 1244-1254, doi:10.1002/hep.23441.

64. Fraser, J.; Cabodevilla, A.G.; Simpson, J.; Gammoh, N. Interplay of autophagy, receptor tyrosine kinase signalling and endocytic trafficking. Essays Biochem 2017, 61, 597-607, doi:10.1042/EBC20170091.

65. Wei, Y.; Zou, Z.; Becker, N.; Anderson, M.; Sumpter, R.; Xiao, G.; Kinch, L.; Koduru, P.; Christudass, C.S.; Veltri, R.W., et al. EGFR-mediated Beclin 1 phosphorylation in autophagy suppression, tumor progression, and tumor chemoresistance. Cell 2013, 154, 1269-1284, doi:10.1016/j.cell.2013.08.015.

66. Yogalingam, G.; Pendergast, A.M. Abl kinases regulate autophagy by promoting the trafficking and function of lysosomal components. J Biol Chem 2008, 283, 35941-35953, doi:10.1074/jbc.M804543200.

67. Takeuchi, H.; Kanzawa, T.; Kondo, Y.; Kondo, S. Inhibition of platelet-derived growth factor signalling induces autophagy in malignant glioma cells. Br J Cancer 2004, 90, 1069-1075, doi:10.1038/sj.bjc.6601605.

68. Lachmann, A.; Torre, D.; Keenan, A.B.; Jagodnik, K.M.; Lee, H.J.; Wang, L.; Silverstein, M.C.; Ma'ayan, A. Massive mining of publicly available RNA-seq data from human and mouse. Nat Commun 2018, 9, 1366, doi:10.1038/s41467-018-03751-6.

69. Uhlen, M.; Fagerberg, L.; Hallstrom, B.M.; Lindskog, C.; Oksvold, P.; Mardinoglu, A.; Sivertsson, A.; Kampf, C.; Sjostedt, E.; Asplund, A., et al. Tissue-based map of the human proteome. Science 2015, 347, 1260419, doi:10.1126/science.1260419. 
70. Kanehisa, M.; Goto, S.; Sato, Y.; Kawashima, M.; Furumichi, M.; Tanabe, M. Data, information, knowledge and principle: back to metabolism in KEGG. Nucleic Acids Res 2014, 42, D199-205, doi:10.1093/nar/gkt1076.

71. Marino, G.; Niso-Santano, M.; Baehrecke, E.H.; Kroemer, G. Self-consumption: the interplay of autophagy and apoptosis. Nat Rev Mol Cell Biol 2014, 15, 81-94, doi:10.1038/nrm3735.

72. Radogna, F.; Dicato, M.; Diederich, M. Cancer-type-specific crosstalk between autophagy, necroptosis and apoptosis as a pharmacological target. Biochem Pharmacol 2015, 94, 1-11, doi:10.1016/j.bcp.2014.12.018.

73. Liu, J.; Kuang, F.; Kroemer, G.; Klionsky, D.J.; Kang, R.; Tang, D. Autophagy-dependent ferroptosis: machinery and regulation. Cell Chem Biol 2020, 10.1016/j.chembiol.2020.02.005, doi:10.1016/j.chembiol.2020.02.005.

74. Kast, D.J.; Dominguez, R. The cytoskeleton-autophagy connection. Curr Biol 2017, 27, R318-R326, doi:10.1016/j.cub.2017.02.061.

75. Menzies, F.M.; Fleming, A.; Caricasole, A.; Bento, C.F.; Andrews, S.P.; Ashkenazi, A.; Fullgrabe, J.; Jackson, A.; Jimenez Sanchez, M.; Karabiyik, C., et al. Autophagy and neurodegeneration: pathogenic mechanisms and therapeutic opportunities. Neuron 2017, 93, 1015-1034, doi:10.1016/j.neuron.2017.01.022.

76. Yamamoto, S.; Kuramoto, K.; Wang, N.; Situ, X.; Priyadarshini, M.; Zhang, W.; Cordoba-Chacon, J.; Layden, B.T.; He, C. Autophagy differentially regulates insulin production and insulin sensitivity. Cell Rep 2018, 23, 3286-3299, doi:10.1016/j.celrep.2018.05.032.

77. Ma, D.; Li, S.; Molusky, M.M.; Lin, J.D. Circadian autophagy rhythm: a link between clock and metabolism? Trends Endocrinol Metab 2012, 23, 319-325, doi:10.1016/j.tem.2012.03.004.

78. Hansen, M.; Rubinsztein, D.C.; Walker, D.W. Autophagy as a promoter of longevity: insights from model organisms. Nat Rev Mol Cell Biol 2018, 19, 579-593, doi:10.1038/s41580-018-0033-y.

79. Qomaladewi, N.P.; Kim, M.Y.; Cho, J.Y. Rottlerin reduces cAMP/CREB-mediated melanogenesis via regulation of autophagy. Int J Mol Sci 2019, 20, doi:10.3390/ijms20092081.

80. Amaravadi, R.K.; Kimmelman, A.C.; Debnath, J. Targeting autophagy in cancer: recent advances and future directions. Cancer Discov 2019, 9, 1167-1181, doi:10.1158/2159-8290.CD-19-0292.

81. Dyczynski, M.; Yu, Y.; Otrocka, M.; Parpal, S.; Braga, T.; Henley, A.B.; Zazzi, H.; Lerner, M.; Wennerberg, K.; Viklund, J., et al. Targeting autophagy by small molecule inhibitors of vacuolar protein sorting 34 (Vps34) improves the sensitivity of breast cancer cells to Sunitinib. Cancer Lett 2018, 435, 3243, doi:10.1016/j.canlet.2018.07.028.

82. Levine, B.; Mizushima, N.; Virgin, H.W. Autophagy in immunity and inflammation. Nature 2011, 469, 323-335, doi:10.1038/nature09782.

83. Pahari, S.; Negi, S.; Aqdas, M.; Arnett, E.; Schlesinger, L.S.; Agrewala, J.N. Induction of autophagy through CLEC4E in combination with TLR4: an innovative strategy to restrict the survival of Mycobacterium tuberculosis. Autophagy 2019, 10.1080/15548627.2019.1658436, 1-23, doi:10.1080/15548627.2019.1658436.

84. Homma, K.; Suzuki, K.; Sugawara, H. The Autophagy Database: an all-inclusive information resource on autophagy that provides nourishment for research. Nucleic Acids Res 2011, 39, D986-990, doi:10.1093/nar/gkq995.

85. Turei, D.; Foldvari-Nagy, L.; Fazekas, D.; Modos, D.; Kubisch, J.; Kadlecsik, T.; Demeter, A.; Lenti, K.; Csermely, P.; Vellai, T., et al. Autophagy Regulatory Network - a systems-level bioinformatics resource 
for studying the mechanism and regulation of autophagy. Autophagy 2015, 11, 155-165, doi:10.4161/15548627.2014.994346.

86. Deng, W.; Ma, L.; Zhang, Y.; Zhou, J.; Wang, Y.; Liu, Z.; Xue, Y. THANATOS: an integrative data resource of proteins and post-translational modifications in the regulation of autophagy. Autophagy 2018, 14, 296-310, doi:10.1080/15548627.2017.1402990.

87. Nanduri, R.; Kalra, R.; Bhagyaraj, E.; Chacko, A.P.; Ahuja, N.; Tiwari, D.; Kumar, S.; Jain, M.; Parkesh, R.; Gupta, P. AutophagySMDB: a curated database of small molecules that modulate protein targets regulating autophagy. Autophagy 2019, 15, 1280-1295, doi:10.1080/15548627.2019.1571717.

88. Wang, N.N.; Dong, J.; Zhang, L.; Ouyang, D.; Cheng, Y.; Chen, A.F.; Lu, A.P.; Cao, D.S. HAMdb: a database of human autophagy modulators with specific pathway and disease information. J Cheminform 2018, 10, 34, doi:10.1186/s13321-018-0289-4.

89. Csermely, P.; Korcsmaros, T.; Kiss, H.J.; London, G.; Nussinov, R. Structure and dynamics of molecular networks: a novel paradigm of drug discovery: a comprehensive review. Pharmacol Ther 2013, 138, 333408, doi:10.1016/j.pharmthera.2013.01.016.

90. Ozdemir, E.S.; Halakou, F.; Nussinov, R.; Gursoy, A.; Keskin, O. Methods for Discovering and Targeting Druggable Protein-Protein Interfaces and Their Application to Repurposing. Methods Mol Biol 2019, 1903, 1-21, doi:10.1007/978-1-4939-8955-3_1.

91. Gonen, M. Predicting drug-target interactions from chemical and genomic kernels using Bayesian matrix factorization. Bioinformatics 2012, 28, 2304-2310, doi:10.1093/bioinformatics/bts360.

92. Gundersen, G.W.; Jones, M.R.; Rouillard, A.D.; Kou, Y.; Monteiro, C.D.; Feldmann, A.S.; Hu, K.S.; Ma'ayan, A. GEO2Enrichr: browser extension and server app to extract gene sets from GEO and analyze them for biological functions. Bioinformatics 2015, 31, 3060-3062, doi:10.1093/bioinformatics/btv297.

93. Gyori, B.M.; Bachman, J.A.; Subramanian, K.; Muhlich, J.L.; Galescu, L.; Sorger, P.K. From word models to executable models of signaling networks using automated assembly. Mol Syst Biol 2017, 13, 954, doi:10.15252/msb.20177651.

94. Pillich, R.T.; Chen, J.; Rynkov, V.; Welker, D.; Pratt, D. NDEx: A Community Resource for Sharing and Publishing of Biological Networks. Methods Mol Biol 2017, 1558, 271-301, doi:10.1007/978-1-4939-67834_13.

95. Gatliff, J.; East, D.; Crosby, J.; Abeti, R.; Harvey, R.; Craigen, W.; Parker, P.; Campanella, M. TSPO interacts with VDAC1 and triggers a ROS-mediated inhibition of mitochondrial quality control. Autophagy 2014, 10, 2279-2296, doi:10.4161/15548627.2014.991665.

96. Sridharan, S.; Jain, K.; Basu, A. Regulation of autophagy by kinases. Cancers (Basel) 2011, 3, 2630-2654, doi:10.3390/cancers3022630.

97. Nussinov, R.; Zhang, M.; Tsai, C.J.; Jang, H. Calmodulin and IQGAP1 activation of PI3Kalpha and Akt in KRAS, HRAS and NRAS-driven cancers. Biochim Biophys Acta Mol Basis Dis 2018, 1864, 2304-2314, doi:10.1016/j.bbadis.2017.10.032.

98. Nussinov, R.; Zhang, M.; Tsai, C.J.; Liao, T.J.; Fushman, D.; Jang, H. Autoinhibition in Ras effectors Raf, PI3Kalpha, and RASSF5: a comprehensive review underscoring the challenges in pharmacological intervention. Biophys Rev 2018, 10, 1263-1282, doi:10.1007/s12551-018-0461-0.

99. Rolf, M.G.; Curwen, J.O.; Veldman-Jones, M.; Eberlein, C.; Wang, J.; Harmer, A.; Hellawell, C.J.; Braddock, M. In vitro pharmacological profiling of R406 identifies molecular targets underlying the clinical effects of fostamatinib. Pharmacol Res Perspect 2015, 3, e00175, doi:10.1002/prp2.175. 
100. Huang, G.; Zhang, F.; Ye, Q.; Wang, H. The circadian clock regulates autophagy directly through the nuclear hormone receptor Nr1d1/Rev-erbalpha and indirectly via Cebpb/(C/ebpbeta) in zebrafish. Autophagy 2016, 12, 1292-1309, doi:10.1080/15548627.2016.1183843.

101. Torres-Quiroz, F.; Filteau, M.; Landry, C.R. Feedback regulation between autophagy and PKA. Autophagy 2015.

102. Chen, Y.; Klionsky, D.J. The regulation of autophagy - unanswered questions. J Cell Sci 2011, 124, 161170, doi:10.1242/jcs.064576.

103. Liu, B.; Bhatt, D.; Oltvai, Z.N.; Greenberger, J.S.; Bahar, I. Significance of p53 dynamics in regulating apoptosis in response to ionizing radiation, and polypharmacological strategies. Sci Rep 2014, 4, 6245.

104. Liu, B.; Liu, Q.; Yang, L.; Palaniappan, S.K.; Bahar, I.; Thiagarajan, P.S.; Ding, J.L. Innate immune memory and homeostasis may be conferred through crosstalk between the TLR3 and TLR7 pathways. Sci Signal 2016, 9, ra70, doi:10.1126/scisignal.aac9340.

105. Liu, B.; Gyori, B.; Thiagarajan, P. Statistical model checking based analysis of biological betworks. Automated Reasoning for Systems Biology and Medicine 2019, 10.1007/978-3-030-17297-8_3, 63-92, doi:10.1007/978-3-030-17297-8_3.

106. Liu, B.; Thiagarajan, P.S. Modeling and analysis of biopathways dynamics. J Bioinform Comput Biol 2012, 10, 1231001, doi:10.1142/S0219720012310014.

107. Ueno, T.; Komatsu, M. Autophagy in the liver: functions in health and disease. Nat Rev Gastroenterol Hepatol 2017, 14, 170-184, doi:10.1038/nrgastro.2016.185.

108. Wang, M.M.; Feng, Y.S.; Yang, S.D.; Xing, Y.; Zhang, J.; Dong, F.; Zhang, F. The relationship between autophagy and brain plasticity in neurological diseases. Front Cell Neurosci 2019, 13, 228, doi:10.3389/fncel.2019.00228.

109. Czaja, M.J.; Ding, W.X.; Donohue, T.M., Jr.; Friedman, S.L.; Kim, J.S.; Komatsu, M.; Lemasters, J.J.; Lemoine, A.; Lin, J.D.; Ou, J.H., et al. Functions of autophagy in normal and diseased liver. Autophagy 2013, 9, 1131-1158, doi:10.4161/auto.25063.

110. Schiattarella, G.G.; Hill, J.A. Therapeutic targeting of autophagy in cardiovascular disease. J Mol Cell Cardiol 2016, 95, 86-93, doi:10.1016/j.yjmcc.2015.11.019.

111. Kagan, V.E.; Mao, G.; Qu, F.; Angeli, J.P.; Doll, S.; Croix, C.S.; Dar, H.H.; Liu, B.; Tyurin, V.A.; Ritov, V.B., et al. Oxidized arachidonic and adrenic PEs navigate cells to ferroptosis. Nat Chem Biol 2017, 13, 81-90, doi:10.1038/nchembio.2238.

112. Wenzel, S.E.; Tyurina, Y.Y.; Zhao, J.; St Croix, C.M.; Dar, H.H.; Mao, G.; Tyurin, V.A.; Anthonymuthu, T.S.; Kapralov, A.A.; Amoscato, A.A., et al. PEBP1 wardens ferroptosis by enabling lipoxygenase generation of lipid death signals. Cell 2017, 171, 628-641, doi:10.1016/j.cell.2017.09.044.

113. Kapralov, A.A.; Yang, Q.; Dar, H.H.; Tyurina, Y.Y.; Anthonymuthu, T.S.; Kim, R.; St Croix, C.M.; Mikulska-Ruminska, K.; Liu, B.; Shrivastava, I.H., et al. Redox lipid reprogramming commands susceptibility of macrophages and microglia to ferroptotic death. Nat Chem Biol 2020, 16, 278-290, doi:10.1038/s41589-019-0462-8.

114. Steinman, J.; Epperly, M.; Hou, W.; Willis, J.; Wang, H.; Fisher, R.; Liu, B.; Bahar, I.; McCaw, T.; Kagan, V., et al. Improved total-body irradiation survival by delivery of two radiation mitigators that target distinct cell death pathways. Radiat Res 2018, 189, 68-83, doi:10.1667/RR14787.1.

115. Thermozier, S.; Hou, W.; Zhang, X.; Shields, D.; Fisher, R.; Bayir, H.; Kagam, V.; Yu, J.; Liu, B.; Bahar, I., et al. Anti-ferroptosis drug enhances total body irradiation mitigation by drugs that block apoptosis and necroptosis. Radiat Res 2020, in press. 
116. Lovric, M.; Molero, J.M.; Kern, R. PySpark and RDKit: Moving towards Big Data in Cheminformatics. Mol Inform 2019, 38, e1800082, doi:10.1002/minf.201800082.

117. Kanehisa, M.; Furumichi, M.; Tanabe, M.; Sato, Y.; Morishima, K. KEGG: new perspectives on genomes, pathways, diseases and drugs. Nucleic Acids Res 2017, 45, D353-D361, doi:10.1093/nar/gkw1092.

118. Benjamini, Y.; Drai, D.; Elmer, G.; Kafkafi, N.; Golani, I. Controlling the false discovery rate in behavior genetics research. Behav Brain Res 2001, 125, 279-284, doi:10.1016/s0166-4328(01)00297-2.

(C) 2020 by the authors. Submitted for possible open access publication under the terms and conditions of the Creative Commons Attribution (CC BY) license (http://creativecommons.org/licenses/by/4.0/). 